Article

\title{
Essential Oils from Fruit and Vegetables, Aromatic Herbs, and Spices: Composition, Antioxidant, and Antimicrobial Activities
}

\author{
Soumi De-Montijo-Prieto ${ }^{1}\left(\mathbb{D}\right.$, María del Carmen Razola-Díaz ${ }^{2,3}{ }^{\circledR}$, Ana María Gómez-Caravaca ${ }^{4, *}$, \\ Eduardo Jesús Guerra-Hernandez ${ }^{2} \mathbb{D}$, María Jiménez-Valera ${ }^{1}$, Belén Garcia-Villanova ${ }^{2} \mathbb{D}$, Alfonso Ruiz-Bravo ${ }^{1}$ \\ and Vito Verardo ${ }^{2,3}$ (D) \\ check for \\ 1 Department of Microbiology, Campus of Cartuja, University of Granada, 18071 Granada, Spain; \\ soumidemontijop@ugr.es (S.D.-M.-P.); mjvalera@ugr.es (M.J.-V.); aruizbr@ugr.es (A.R.-B.) \\ 2 Department of Nutrition and Food Science, Campus of Cartuja, University of Granada, 18071 Granada, Spain; \\ carmenrazola@correo.ugr.es (M.d.C.R.-D.); ejguerra@ugr.es (E.J.G.-H.); belenv@ugr.es (B.G.-V.); \\ vitoverardo@ugr.es (V.V.) \\ 3 Institute of Nutrition and Food Technology 'José Mataix', Biomedical Research Center, University of Granada, \\ Avda del Conocimiento sn., Armilla, 18100 Granada, Spain \\ 4 Department of Analytical Chemistry, Faculty of Sciences, University of Granada, Avd. Fuentenueva s/n., \\ 18071 Granada, Spain \\ * Correspondence: anagomez@ugr.es
} updates

Citation: De-Montijo-Prieto, S.;

Razola-Díaz, M.d.C.

Gómez-Caravaca, A.M.;

Guerra-Hernandez, E.J.;

Jiménez-Valera, M.; Garcia-Villanova,

B.; Ruiz-Bravo, A.; Verardo, V.

Essential Oils from Fruit and

Vegetables, Aromatic Herbs, and Spices: Composition, Antioxidant, and Antimicrobial Activities. Biology 2021, 10, 1091. https://doi.org/ 10.3390/biology10111091

Academic Editor:

Francesca Mancianti

Received: 3 August 2021

Accepted: 20 October 2021

Published: 25 October 2021

Publisher's Note: MDPI stays neutral with regard to jurisdictional claims in published maps and institutional affiliations.

Copyright: (C) 2021 by the authors Licensee MDPI, Basel, Switzerland. This article is an open access article distributed under the terms and conditions of the Creative Commons Attribution (CC BY) license (https:/ / creativecommons.org/licenses/by/ $4.0 /)$.
Simple Summary: The use of essential oils (EOs) in the food industry is a popular research topic, as they have antioxidant and antimicrobial activity and could be used as ingredients directly in food or as bioactive component in food coating and food packaging. Thus, the study of their antioxidant and antimicrobial activity is a crucial step to evaluate their use in food packaging/coating. In this work, we evaluate the antioxidant and antimicrobial activities of 13 EOs from herbs, spices, fruits, and vegetables. Briefly, the EOs from aromatic herbs and spices showed the highest antioxidant and antimicrobial activity. Fennel essential oil reported the lowest antioxidant activity, however it showed very good antimicrobial activity against Botrytis cinerea, one of the post-harvest pathogen microorganisms in fruits and vegetables.

Abstract: In the field of food preservation, encapsulated Essential Oils (EOs) could be the best non-toxic and eco-friendly tool for food preservative applications substituting the chemicals ones that have several disadvantages for the environment and health. Thirteen commercial EOs from plants, fruits, and vegetables were characterized by GC-MS. The antioxidant activity was measured by DPPH and ABTS techniques. Antimicrobial activity was assessed by agar well-diffusion method and the Minimum Inhibitory Concentration (MIC) by agar dilution method against six bacteria, Candida albicans, and Botrytis cinerea. All the EOs tested have demonstrated antioxidant activity in the range of $\mathrm{IC}_{50} 0.01-105.32 \mathrm{mg} / \mathrm{mL}$. Between them, cinnamon EOs were the best, followed by oregano and thyme EOs. Fennel EO showed the lowest radical scavenging. MIC values ranged from 0.14 to $9 \mathrm{mg} / \mathrm{mL}$. C. cassia, thyme, and oregano EOs were the most effective against the bacterial species tested, and the yeast $C$. albicans. On the contrary, citric fruit EOs showed low or no inhibition against most bacterial strains. The percentages of inhibition of mycelia growth of B. cinerea ranged from 3.4 to $98.5 \%$. Thyme, oregano, mint, and fennel EOs showed the highest inhibition.

Keywords: essential oils; DPPH; ABTS; food spoilage; antiradical activity

\section{Introduction}

Food security is supported in four main pillars: food access, food utilization, food stability, and food preservation. The latter mainly consists of the degradation and microbial contamination that can affect food. Food spoilage is one of the problems that should be avoided. A lot of chemical preservatives have been developed and proved to have a 
significant contribution in controlling this degradation. However, they have often raised negative concerns to the consumers as they are not from a green source. They need long term degradation cycles, they are environmental toxicology and have potential risks of carcinogenesis and teratogenesis in humans and animals. Due to this, essential oils (EOs) and their active components are under study for their potential use as preservatives due to their wide antibacterial, antifungal, antimycotoxigenic spectrum, and antioxidant properties [1]. Therefore, the use of EOs in food industry is growing, as they could be directly added to edible products or used for active packaging and edible coatings [2] Thus, the use of essential oil in food industry has a double action due to their antioxidant and antimicrobial properties [3]. Moreover, the bioactive compounds contained in the EOs could also be used for pharmaceutical and cosmetic applications [4].

Overall, EOs are products that can be isolated from leaves, bark, seeds, fruit peels, roots, flowers, buds, and stems, namely, from agro-industrial by-products in the majority of cases. The antioxidant activity of the EOs is related to the complex mixture of terpenes, terpenoids, and phenylpropanoids that compose them. Among others, it was noticed that carvacrol, thymol, and eugenol are able to inhibit the oxidation processes [5].

The same happens with the antimicrobial activity of the EOs, but in this case the mechanism depends on the specific chemical components. The most common mechanism seems to be related with the alteration in the membranes, modifying their dynamicity and permeability, and in consequence releasing the cytoplasmic constituents. However, the effect is different for each microorganism depending on the variability of the membrane thickness, composition, and cellular metabolic activities [1].

The limitations that could carry the use of EOs as preservatives in food can be due to the intense aroma, high reactivity, hydrophobicity, reduced solubility, and possible negative interaction with the matrices, leading to changes in the intestinal absorbance and organoleptic properties. However, nowadays they are avoidable due to the new mechanisms studied and developed for encapsulating those EOs. These methods can improve the stability and solubility of the EOs, protecting them from the environmental interactions [6].

Therefore, for agriculture and food fields, encapsulated EOs could be the best non-toxic and eco-friendly option for preservative applications as active food packaging, enhancing food shelf life. In this context, one of the goals of the SHEALTHY project is to find essential oils that could be incorporated in food packaging in order to obtain active packaging/coating for fruit and vegetables. In this way, it is important to identify essential oils with antibacterial and anti-fungus properties that can act against foodborne pathogens, e.g., Botrytis cinerea, a necrotrophic fungus that affects several fruits, causing the production of high quantities of fruit wastes. Thus, the aim of this work is to evaluate and compare the antioxidant and antimicrobial activities of 13 commercial EOs from plants and fruit and vegetables. To achieve this, the EOs were characterized by GC-MS. The antioxidant activity was evaluated by DPPH (2,2-diphenyl-1-picrylhydrazyl) and ABTS (2,2'-Azinobis (3-ethylbenzothiazoline-6-sulfonic acid) diammonium salt) assays. The antimicrobial activity was tested against microorganisms that usually spoil food and are dangerous for human health.

\section{Materials and Methods}

\subsection{Reagents and Samples}

All reagents were purchased from Sigma-Aldrich (St. Louis, MO, USA). Solvents were from Honeywell (Wabash, Lafayette, IN, USA) except ethanol, which was from PanReac (Barcelona, Spain). Water was purified using a Milli-Q system (Millipore, Bedford, MA, USA).

Thirteen essential oils were purchased from a local supplier; seven of them hail from aromatic plants and spices, and others were from fruits and vegetables (Table 1). All samples were obtained by hydrodistillation (purity 100\%). Selection of the essential oils took the literature data and the availability in the Spanish market into account. 
Table 1. List of the EOs used in this work.

\begin{tabular}{ccc}
\hline Common Name & Scientific Name & Part Used \\
\hline True cinnamon & Aromatic herbs and spices & \\
Cinnamon & Cinnamomum zeylanicum J. Presl & Leaf \\
Oregano & Cinnamomum cassia J. Presl & Bark \\
Thyme & Origanum vulgare L. & Leaf \\
Rosemary & Thymus vulgaris L. & Flower/leaf \\
Peppermint & Rosmarinus officinalis (L.) Schleid. & Leaf \\
Sage & Mentha piperita L. & Leaf \\
& Salvia lavandulifolia Vahl & Leaf \\
\hline Celery & Fruits and vegetables & \\
Fennel & Apium graveolens L. & Seed \\
Mandarin & Foeniculum vulgare Mill. var. dulce & Not reported \\
Sweet orange & Citrus reticulata Blanco & Peel \\
Lemon & Citrus sinensis Osbeck & Peel \\
Grapefruit & Citrus limon L. & Peel \\
\hline & Citrus paradisi Macfad. & Peel \\
\hline
\end{tabular}

\subsection{DPPH Free Radical-Scavenging Capacity}

DPPH radical scavenging activity was assayed with a method proposed by several authors [7,8]. Briefly, $2.9 \mathrm{~mL}$ of $100 \mu \mathrm{M} \mathrm{DPPH}$ (in methanol or $n$-hexane depending on the polarity of the essential oil) was mixed with $100 \mu \mathrm{L}$ of each essential oil at different concentrations. They were incubated during $30 \mathrm{~min}$ at $25^{\circ} \mathrm{C}$ and were measured at $517 \mathrm{~nm}$. The antioxidant activity of the EOs was expressed as the concentration of extract that inhibited the DPPH radical formation by $50 \%\left(\mathrm{IC}_{50}\right) . \mathrm{IC}_{50}$ for each sample was calculated by elaborating a curve where is represented the concentration $(\mathrm{mg} / \mathrm{mL})$ and the percentage of inhibition calculated as in the following Equation (1):

$$
\text { Inhibition }(\%)=(\text { blank }- \text { sample }) / \text { blank } \times 100
$$

where "blank" is the absorbance of DPPH with the sample replaced with methanol and "sample" is referred to the absorbance of the DPPH mixed with the essential oil or the control. Ascorbic acid was used as a reference of positive control from 0.0001 to $0.5 \mathrm{mg} / \mathrm{mL}$.

\subsection{ABTS Acid Cation Radical-Scavenging Capacity}

This technique was developed by Re at al. [9] in which the monocation ABTS ${ }^{\bullet+}$ is generated by oxidation of the ABTS with potassium persulfate in the dark at room temperature for 12-24 h. For the analyses, $2 \mathrm{~mL}$ of $7 \mathrm{mM}$ ABTS solution was added to $20 \mu \mathrm{L}$ of different concentrations of each sample, and they were measured at $734 \mathrm{~nm}$ after $30 \mathrm{~min}$ of incubation at $30^{\circ} \mathrm{C}$. The antioxidant activity of the EOs was expressed as the concentration of extract that inhibited the ABTS radical formation by $50 \%\left(\mathrm{IC}_{50}\right)$. The $\mathrm{IC}_{50}$ for each sample was calculated by elaborating a curve where is represented the concentration $(\mathrm{mg} / \mathrm{mL})$ and the percentage of inhibition calculated as in the Equation (1). Ascorbic acid was used as a reference of positive control from 0.0001 to $0.5 \mathrm{mg} / \mathrm{mL}$.

\subsection{Antimicrobial Activity}

\subsubsection{Test Microorganisms}

The antimicrobial activity of essential oils was tested against Staphylococcus aureus (S. aureus), methicillin-resistant Staphylococcus aureus (MRSA), Escherichia coli (E. coli), Salmonella enterica serovar Typhimurium (S. Typhimurium), Listeria monocytogenes (L. monocytogenes), Candida albicans (C. albicans), and Botrytis cinerea (B. cinerea). All bacterial strains and $C$. albicans were isolated from various clinical samples in the Microbiology Service of the Virgen de las Nieves Hospital (Granada, Spain) and were stored as glycerol stocks and reactivated by incubation in tryptic soy agar at $37^{\circ} \mathrm{C}$ for $24 \mathrm{~h}$. B. cinerea was 
obtained from the Spanish Type Culture Collection (CECT 2100) and was maintained and grown at $25^{\circ} \mathrm{C}$ in Sabouraud dextrose agar.

\subsubsection{Agar-Well-Diffusion Method}

For bacteria and C. albicans, antimicrobial activity was assessed following the method described by Hayes and Markovic [10] with modifications, as follows: $15 \mathrm{~mL}$ of molten Mueller Hinton agar were poured into sterile Petri dishes and allowed to set to form a base layer. An $8 \mathrm{~mm}$ diameter stainless steel cylinder was placed over the base layer, and $10 \mathrm{~mL}$ of molten Mueller Hinton agar containing the inoculum were poured over the surface of the base layer and left to set. For the preparation of inoculate, cultures of the strains were suspended in buffered saline solution until reaching a turbidity corresponding to 0.5 McFarland standard and were inoculated in molten Mueller Hinton agar to obtain a final concentration of approximately $1 \times 10^{6} \mathrm{CFU} / \mathrm{mL}$. After solidification of the upper layer, the cylinders were carefully removed and $25 \mu \mathrm{L}$ of essential oils were added into the resulting holes. As a positive control for antimicrobial activity, ciprofloxacin was used at $2 \mathrm{mg} / \mathrm{mL}$ for MRSA, $0.1 \mathrm{mg} / \mathrm{mL}$ for L. monocytogenes, and $0.01 \mathrm{mg} / \mathrm{mL}$ for the remaining bacteria, and ketoconazole at $0.01 \mathrm{mg} / \mathrm{mL}$ for $\mathrm{C}$. albicans. After incubation at $4{ }^{\circ} \mathrm{C}$ for $30 \mathrm{~min}$ to allow extracts to diffuse into the medium, the plates were incubated at $37^{\circ} \mathrm{C}$ for $24 \mathrm{~h}$. The inhibition zone diameters were measured $(\mathrm{mm})$ and recorded as the mean \pm standard deviation (SD). Three replicates were conducted.

For B. cinerea, Sabouraud dextrose plates were prepared following the previous procedure but testing one extract per plate. After $30 \mathrm{~min}$ incubation to allow the extract to diffuse, a 4-mm plug of mycelium was placed at a $3 \mathrm{~cm}$ distance from the essential oil. The plates were incubated for 7 days at $25^{\circ} \mathrm{C}$. The growth control consisted of Sabouraud dextrose plates inoculated with mycelium alone. Ketoconazole at $20 \mu \mathrm{g} / \mathrm{mL}$ was used as antimicrobial control. The percentage of mycelium inhibition for each essential oil was calculated by measuring the area of fungal growth and comparing it to the control using the ImageJ software [11]. Three replicates were conducted.

\subsubsection{Determination of Minimum Inhibitory Concentration (MIC)}

MIC values were assessed by agar-dilution method as follows: from $288 \mathrm{mg} / \mathrm{mL}$ solutions in $98 \%$ ethanol of each essential oil, serial dilutions in sterile water supplemented with $0.5 \%$ Tween 80 were prepared $(144-0.56 \mathrm{mg} / \mathrm{mL})$. A total of $5 \mathrm{~mL}$ of the essential oil's dilutions were added in $15 \mathrm{~mL}$ of molten Mueller Hinton or Sabouraud agar, which was prepared previously with three-quarters of its volume of water, and plated onto sterile Petri dishes. The essential oils were tested in concentrations ranging from 72 to $0.14 \mathrm{mg} / \mathrm{mL}$ for bacteria and C. albicans, and from 72 to $0.07 \mathrm{mg} / \mathrm{mL}$ for B. cinerea. Bacterial suspensions corresponding to $0.5 \mathrm{McFarland}$ 's standard were adjusted to obtain a concentration of approximately $1 \times 10^{6} \mathrm{CFU} / \mathrm{mL}$, and a spot of $10 \mu \mathrm{L}$ of each bacterial suspension was added onto the plates. B. cinerea was inoculated placing a 4-mm plug of mycelium at the center of each plate. A plate containing $5 \mathrm{~mL}$ of sterile water supplemented with $0.5 \%$ Tween 80 in $15 \mathrm{~mL}$ of molten Mueller Hinton or Sabouraud agar prepared and inoculated as before, was used as a growth control. All experiments were performed in duplicate. The MIC was defined as the lowest concentration of the essential oils that completely inhibited microbial growth after incubation at $37^{\circ} \mathrm{C}$ for $24 \mathrm{~h}$ for bacteria and C. albicans, and $25^{\circ} \mathrm{C}$ for 7 days for B. cinerea.

\subsection{Determination of Essential Oil Compounds by GC-MS}

Essential oils were diluted in trichloromethane and analysed by GC-MS according to Ben Lajnef et al. [12]. Separation was achieved using an Agilent 7890A GC coupled to a Waters QUATTRO micro ${ }^{\mathrm{TM}}$ GC mass spectrometer. The compounds were separated on a capillary column DB-5MS $(30 \mathrm{~m} \times 0.25 \mathrm{~mm}$; f.t. $0.25 \mu \mathrm{m})$ purchased from Agilent Technologies (J\&W Scientific, Folsom, CA, USA). Oven temperature was set at $40{ }^{\circ} \mathrm{C}$ for 
$2 \mathrm{~min}$, after that the temperature increased from 40 to $250{ }^{\circ} \mathrm{C}$ at $3{ }^{\circ} \mathrm{C} \mathrm{min}^{-1}$, and remained at $250{ }^{\circ} \mathrm{C}$ for $10 \mathrm{~min}$.

MS detector parameters were set at: scan range: $40-450 \mathrm{~m} / \mathrm{z}$; solvent delay time: $3.0 \mathrm{~min}$; transfer line temperature $250^{\circ} \mathrm{C}$; ion source $230^{\circ} \mathrm{C}$; and ionisation energy $70 \mathrm{eV}$. Carrier gas was $(\mathrm{He})$ at a flow of $1.0 \mathrm{~mL} \mathrm{~min}{ }^{-1}$. Samples were injected in splitless mode. GS-MS chromatograms are showed in Supplementary Figure S1.

\subsection{Statistical Analysis}

Pearson's correlations between antioxidant methods were evaluated using Statistica 6.0 (2001, StatSoft, Tulsa, OK, USA).

\section{Results and Discussion}

\subsection{Composition of the Essential Oils}

Essential oils were analyzed by GC-MS and a total of 56 compounds were detected. Table 2 reported the relative amounts (\%) of each compound determined in aromatic plant EOs.

According to the literature [13], cinnamon samples presented different composition according to the part of the plant that was used for the extraction. Briefly, eugenol was the main compound in C. zeylanicum that was extracted from leaves; on the other hand, cinnamaldehyde was the first compound in C. cassia sample that was extracted from bark.

Oregano EO showed carvacrol as the main compound (84.5\%) followed by $p$-cymene, $\gamma$ terpinene and thymol. Similar composition has been reported by Diniz do Nascimento et al. [4]; as remarked by the same authors, the oregano essential oil composition is highly influenced by the part of the plant and the agronomic and technological processes.

Thymus EO composition is also affected from the different parts of plant that are used for extraction (among agronomical and processing factors). The samples that were analyzed in this work proceed from a mix of flowers and leaves; thymol was the first compound accounting for more than $60 \%$, followed by $p$-cymene, $\gamma$-terpinene, carvacrol, and linalool, respectively, accounting for about the $90 \%$ of total compound. The same compounds were described by Diniz do Nascimento et al. [4] in thymus EOs.

$\beta$-myrcene was the first compound in Rosmarinus EO, the second one was camphor, followed by eucalyptol and $\alpha$-pinene; the present composition is in the same order of magnitude than that reported by Diniz do Nascimento et al. [4].

Mentha EO reported the typical compounds previously found in this matrix [4] and are characteristic of this EO. Briefly, menthol and menthone accounted for $69.5 \%$ of the total compounds, followed by menthyl-acetate, eucalyptol, isomenthone, isomenthol, and mentho-furan. Other minor compounds that are usually found in this plant are piperitone and neomenthol acetate.

Finally, according to Porres-Martínez et al. [14], 1,8-cineole (eucalyptol) followed by camphor, camphene, and $\beta$ - and $\alpha$-pinene were the main compounds of sage EO.

Table 3 reports the composition of essential oil obtained from fruits and vegetables. 
Table 2. Composition (expressed as \%) of aromatic plant EOs determined by GC-MS.

\begin{tabular}{|c|c|c|c|c|c|c|c|c|}
\hline & Compound & $\begin{array}{l}\text { Cinnamomum } \\
\text { cassia }\end{array}$ & $\begin{array}{c}\text { Cinnamomum } \\
\text { zeylanicum }\end{array}$ & $\begin{array}{l}\text { Mentha } \\
\text { piperita }\end{array}$ & $\begin{array}{c}\text { Origanum } \\
\text { vulgare }\end{array}$ & $\begin{array}{l}\text { Rosmarinus } \\
\text { officinalis }\end{array}$ & $\begin{array}{c}\text { Salvia } \\
\text { lavandulifolia }\end{array}$ & $\begin{array}{l}\text { Thymus } \\
\text { vulgaris }\end{array}$ \\
\hline 1 & Tricyclene & n.d. & n.d. & n.d. & n.d. & $0.2 \pm 0.02$ & $0.2 \pm 0.01$ & $0.08 \pm 0.00$ \\
\hline 2 & Thujene & n.d. & $0.04 \pm 0.00$ & $0.04 \pm 0.00$ & $0.3 \pm 0.01$ & $0.2 \pm 0.00$ & $0.2 \pm 0.01$ & $0.6 \pm 0.03$ \\
\hline 4 & Camphene & n.d. & $0.08 \pm 0.00$ & $0.06 \pm 0.00$ & $0.2 \pm 0.00$ & $5.0 \pm 0.30$ & $7.7 \pm 0.40$ & $0.7 \pm 0.01$ \\
\hline 5 & Sabinene & n.d. & n.d. & $0.2 \pm 0.00$ & n.d. & n.d. & $1.1 \pm 0.30$ & $0.004 \pm 0.00$ \\
\hline 6 & $\beta$-pinene & $0.03 \pm 0.00$ & $0.07 \pm 0.00$ & $0.9 \pm 0.02$ & $0.07 \pm 0.01$ & $2.5 \pm 0.30$ & $5.1 \pm 0.20$ & $0.1 \pm 0.01$ \\
\hline 7 & $\beta$-myrcene & n.d. & $0.008 \pm 0.00$ & n.d. & $0.5 \pm 0.30$ & $30.7 \pm 0.40$ & $2.0 \pm 0.08$ & $0.7 \pm 0.03$ \\
\hline 9 & $\alpha$-terpinene & $0.02 \pm 0.00$ & $0.01 \pm 0.00$ & $0.1 \pm 0.00$ & $0.5 \pm 0.02$ & $0.6 \pm 0.02$ & $0.3 \pm 0.01$ & $1.2 \pm 0.10$ \\
\hline 10 & $p$-cymene & $0.05 \pm 0.00$ & $0.1 \pm 0.01$ & $0.3 \pm 0.03$ & $5.3 \pm 0.20$ & $1.6 \pm 0.10$ & $0.3 \pm 0.02$ & $16.5 \pm 0.30$ \\
\hline 11 & D-limonene & $1.5 \pm 0.20$ & $0.09 \pm 0.00$ & $2.1 \pm 0.20$ & $0.2 \pm 0.01$ & $2.9 \pm 0.30$ & $4.7 \pm 0.40$ & $0.3 \pm 0.02$ \\
\hline 12 & 1,8-cineole (Eucalyptol) & n.d. & n.d. & $4.9 \pm 0.30$ & $0.07 \pm 0.00$ & $14.8 \pm 0.50$ & $38.6 \pm 0.80$ & n.d. \\
\hline 13 & cis-ocimene & n.d. & n.d. & n.d. & n.d. & $0.1 \pm 0.00$ & $0.06 \pm 0.00$ & n.d. \\
\hline 14 & $\gamma$-terpinene & n.d. & n.d. & $0.1 \pm 0.03$ & $2.4 \pm 0.20$ & $0.7 \pm 0.02$ & $0.4 \pm 0.01$ & $7.1 \pm 0.30$ \\
\hline 15 & Terpinolene & n.d. & $0.02 \pm 0.00$ & $0.05 \pm 0.00$ & $0.03 \pm 0.00$ & $0.3 \pm 0.03$ & $0.2 \pm 0.00$ & $0.05 \pm 0.00$ \\
\hline 16 & Linalool & $1.8 \pm 0.10$ & $0.4 \pm 0.01$ & n.d. & $0.7 \pm 0.04$ & $0.5 \pm 0.06$ & $0.7 \pm 0.00$ & $2.5 \pm 0.08$ \\
\hline 17 & Pinone & n.d. & n.d. & $0.004 \pm 0.00$ & n.d. & n.d. & $0.3 \pm 0.01$ & n.d. \\
\hline 19 & Menthone & n.d. & n.d. & $29.8 \pm 0.50$ & n.d. & n.d. & n.d. & n.d. \\
\hline 20 & Menthofuran & $0.002 \pm 0.00$ & n.d. & $1.9 \pm 0.30$ & n.d. & $0.004 \pm 0.00$ & n.d. & n.d. \\
\hline 21 & Isomenthone & $0.002 \pm 0.00$ & $0.002 \pm 0.00$ & $4.2 \pm 0.20$ & n.d. & $0.01 \pm 0.00$ & n.d. & n.d. \\
\hline 22 & Isomenthol & n.d. & n.d. & $3.6 \pm 0.10$ & n.d. & n.d. & n.d. & n.d. \\
\hline 23 & Borneol & n.d. & $0.004 \pm 0.00$ & n.d. & $0.3 \pm 0.04$ & $0.9 \pm 0.10$ & $2.0 \pm 0.20$ & $1.3 \pm 0.10$ \\
\hline 24 & Menthol & n.d. & n.d. & $39.7 \pm 0.30$ & n.d. & n.d. & n.d. & n.d. \\
\hline 25 & Terpinen-4-ol & n.d. & $0.02 \pm 0.00$ & n.d. & $0.3 \pm 0.00$ & $0.4 \pm 0.01$ & $0.4 \pm 0.02$ & $1.0 \pm 0.1$ \\
\hline 26 & $\alpha$-terpineol & n.d. & $0.04 \pm 0.00$ & $0.2 \pm 0.00$ & n.d. & $1.0 \pm 0.06$ & $0.4 \pm 0.01$ & $0.1 \pm 0.01$ \\
\hline 27 & Estragole & n.d. & n.d. & n.d. & n.d. & n.d. & n.d. & $0.01 \pm 0.00$ \\
\hline 28 & Verbenone & $0.006 \pm 0.00$ & n.d. & n.d. & n.d. & $0.3 \pm 0.00$ & n.d. & $0.01 \pm 0.00$ \\
\hline 29 & Methyl thymyl ether & n.d. & n.d. & n.d. & n.d. & n.d. & n.d. & $0.1 \pm 0.02$ \\
\hline 30 & Pulegone & n.d. & n.d. & $0.8 \pm 0.10$ & n.d. & n.d. & n.d. & n.d. \\
\hline 31 & Linalyl anthranilate & n.d. & n.d. & n.d. & n.d. & n.d. & $2.2 \pm 0.30$ & n.d. \\
\hline 32 & Piperitone & n.d. & n.d. & $0.5 \pm 0.03$ & n.d. & n.d. & n.d. & n.d. \\
\hline 33 & Neomenthol acetate & $0.003 \pm 0.00$ & n.d. & $0.3 \pm 0.01$ & n.d. & n.d. & n.d. & n.d. \\
\hline 34 & Cinnamaldehyde & $91.9 \pm 1.70$ & n.d. & n.d. & n.d. & n.d. & n.d. & n.d. \\
\hline 35 & Bornyl acetate & n.d. & n.d. & n.d. & n.d. & $0.8 \pm 0.02$ & $0.8 \pm 0.01$ & n.d. \\
\hline 36 & Myrtenyl acetate & n.d. & n.d. & n.d. & n.d. & n.d. & $1.1 \pm 0.02$ & n.d. \\
\hline
\end{tabular}


Table 2. Cont.

\begin{tabular}{|c|c|c|c|c|c|c|c|c|}
\hline & Compound & $\begin{array}{l}\text { Cinnamomum } \\
\text { cassia }\end{array}$ & $\begin{array}{c}\text { Cinnamomum } \\
\text { zeylanicum }\end{array}$ & $\begin{array}{l}\text { Mentha } \\
\text { piperita }\end{array}$ & $\begin{array}{c}\text { Origanum } \\
\text { vulgare }\end{array}$ & $\begin{array}{c}\text { Rosmarinus } \\
\text { officinalis }\end{array}$ & $\begin{array}{c}\text { Salvia } \\
\text { lavandulifolia }\end{array}$ & $\begin{array}{l}\text { Thymus } \\
\text { vulgaris }\end{array}$ \\
\hline 37 & Menthyl acetate & n.d. & n.d. & $6.1 \pm 0.70$ & n.d. & n.d. & n.d. & n.d. \\
\hline 38 & $\beta$-isosafrole & n.d. & $0.1 \pm 0.00$ & n.d. & n.d. & n.d. & n.d. & n.d. \\
\hline 40 & Carvacrol & n.d. & n.d. & n.d. & $84.5 \pm 1.60$ & n.d. & n.d. & $2.6 \pm 0.5$ \\
\hline 41 & Terpinyl acetate & n.d. & n.d. & n.d. & n.d. & n.d. & $0.6 \pm 0.02$ & $0.06 \pm 0.00$ \\
\hline 42 & Eugenol & $4.2 \pm 0.30$ & $95.2 \pm 1.40$ & n.d. & n.d. & n.d. & n.d. & n.d. \\
\hline 43 & $\beta$-caryophyllene & $0.5 \pm 0.02$ & $1.6 \pm 0.20$ & $2.7 \pm 0.10$ & $1.6 \pm 0.10$ & $2.2 \pm 0.20$ & $0.7 \pm 0.10$ & $1.9 \pm 0.20$ \\
\hline 45 & $\beta$-eudesmene & n.d. & n.d. & $0.08 \pm 0.00$ & n.d. & n.d. & n.d. & $0.07 \pm 0.00$ \\
\hline 46 & Eremophilane & n.d. & n.d. & $0.03 \pm 0.00$ & n.d. & n.d. & $0.2 \pm 0.01$ & $0.01 \pm 0.00$ \\
\hline 47 & Bisabolene & n.d. & n.d. & n.d. & 0.12 & n.d. & n.d. & $0.07 \pm 0.00$ \\
\hline 48 & Isoeugenol & n.d. & $0.4 \pm 0.10$ & $0.2 \pm 0.00$ & n.d. & $0.3 \pm 0.03$ & $0.1 \pm 0.00$ & $0.07 \pm 0.00$ \\
\hline 49 & 2-(2-propenyl)-furan & n.d. & $0.007 \pm 0.00$ & n.d. & n.d. & n.d. & n.d. & n.d. \\
\hline \multirow[t]{9}{*}{50} & Benzyl benzoate & n.d. & $1.0 \pm 0.30$ & n.d. & n.d. & n.d. & n.d. & n.d. \\
\hline & Monoterpenes & 3.4 & 1.3 & 39.9 & 11.4 & 58.2 & 28.9 & 30.8 \\
\hline & $\begin{array}{l}\text { Oxygenated } \\
\text { monoterpenes }\end{array}$ & n.d. & n.d. & 5.0 & 0.1 & 35.5 & 62.2 & 0.6 \\
\hline & Ethers & n.d. & n.d. & n.d. & n.d. & n.d. & n.d. & 0.1 \\
\hline & Esthers & n.d. & 1.0 & 6.4 & n.d. & 0.8 & 2.5 & 0.1 \\
\hline & $\begin{array}{l}\text { Sesquiterpene } \\
\text { hydrocarbons }\end{array}$ & 0.5 & 1.8 & 3.1 & 1.8 & 2.9 & 1.3 & 2.1 \\
\hline & Aldehydes & 91.9 & n.d. & n.d. & n.d. & n.d. & n.d. & n.d. \\
\hline & Phenols & 4.2 & 95.7 & 0.2 & 86.1 & 0.3 & 0.1 & 63.9 \\
\hline & Others & n.d. & 0.2 & 1.9 & n.d. & n.d. & 2.2 & n.d. \\
\hline
\end{tabular}

n.d. $=$ not detected

Table 3. Composition (expressed as \%) of fruit and vegetable EOs determined by GC-MS.

\begin{tabular}{|c|c|c|c|c|c|c|c|}
\hline & Compound & $\begin{array}{c}\text { Apium } \\
\text { graveolens }\end{array}$ & $\begin{array}{l}\text { Citrus } \\
\text { limon }\end{array}$ & $\begin{array}{c}\text { Citrus } \\
\text { paradisi }\end{array}$ & $\begin{array}{c}\text { Citrus } \\
\text { reticulata }\end{array}$ & $\begin{array}{c}\text { Citrus } \\
\text { sinensis }\end{array}$ & $\begin{array}{c}\text { Foeniculum } \\
\text { vulgare }\end{array}$ \\
\hline 1 & Tricyclene & n.d. & n.d. & n.d. & $0.003 \pm 0.00$ & n.d. & $0.004 \pm 0.00$ \\
\hline 2 & Thujene & $0.009 \pm 0.00$ & $0.1 \pm 0.02$ & n.d. & $0.01 \pm 0.00$ & n.d. & n.d. \\
\hline
\end{tabular}


Table 3. Cont.

\begin{tabular}{|c|c|c|c|c|c|c|c|}
\hline & Compound & $\begin{array}{c}\text { Apium } \\
\text { graveolens }\end{array}$ & $\begin{array}{l}\text { Citrus } \\
\text { limon }\end{array}$ & $\begin{array}{c}\text { Citrus } \\
\text { paradisi }\end{array}$ & $\begin{array}{c}\text { Citrus } \\
\text { reticulata }\end{array}$ & $\begin{array}{c}\text { Citrus } \\
\text { sinensis }\end{array}$ & $\begin{array}{c}\text { Foeniculum } \\
\text { vulgare }\end{array}$ \\
\hline 3 & $\alpha$-pinene & $0.3 \pm 0.04$ & $0.8 \pm 0.05$ & $0.1 \pm 0.00$ & $0.2 \pm 0.02$ & $0.2 \pm 0.03$ & $1.2 \pm 0.10$ \\
\hline 4 & Camphene & n.d. & $0.03 \pm 0.00$ & n.d. & n.d. & n.d. & $0.01 \pm 0.00$ \\
\hline 5 & Sabinene & $0.06 \pm 0.00$ & $0.8 \pm 0.10$ & $0.2 \pm 0.01$ & $0.2 \pm 0.00$ & $0.2 \pm 0.00$ & $0.03 \pm 0.00$ \\
\hline 6 & $\beta$-pinene & $2.4 \pm 0.20$ & $6.0 \pm 0.30$ & $0.08 \pm 0.00$ & $0.05 \pm 0.00$ & $0.05 \pm 0.00$ & $0.02 \pm 0.00$ \\
\hline 7 & $\beta$-myrcene & $0.4 \pm 0.02$ & $0.4 \pm 0.10$ & $0.3 \pm 0.03$ & $0.4 \pm 0.02$ & $0.4 \pm 0.01$ & $0.01 \pm 0.00$ \\
\hline 8 & $\alpha$-terpinene & n.d. & $0.08 \pm 0.00$ & n.d. & n.d. & n.d. & $0.003 \pm 0.00$ \\
\hline 9 & Cymene & $0.06 \pm 0.00$ & $0.1 \pm 0.02$ & $0.02 \pm 0.00$ & $0.03 \pm 0.00$ & $0.02 \pm 0.00$ & $0.01 \pm 0.00$ \\
\hline 10 & D-limonene & $71.4 \pm 1.50$ & $87.0 \pm 1.20$ & $99.0 \pm 1.60$ & $99.0 \pm 1.10$ & $99.2 \pm 1.50$ & $1.2 \pm 0.40$ \\
\hline 11 & 1,8-cineole (Eucalyptol) & n.d. & n.d. & n.d. & n.d. & n.d. & $0.01 \pm 0.00$ \\
\hline 12 & $\gamma$-terpinene & n.d. & $3.8 \pm 0.07$ & $0.04 \pm 0.00$ & $0.06 \pm 0.00$ & n.d. & $0.01 \pm 0.00$ \\
\hline 13 & Terpinolene & n.d. & $0.1 \pm 0.02$ & n.d. & n.d. & n.d. & $0.03 \pm 0.00$ \\
\hline 14 & Fenchone & $0.003 \pm 0.00$ & n.d. & n.d. & $0.002 \pm 0.00$ & n.d. & $0.3 \pm 0.01$ \\
\hline 15 & Linalool & n.d. & n.d. & n.d. & n.d. & n.d. & $0.001 \pm 0.00$ \\
\hline 16 & Pinone & $0.01 \pm 0.00$ & $0.001 \pm 0.00$ & n.d. & $0.002 \pm 0.00$ & n.d. & $0.0005 \pm 0.00$ \\
\hline 17 & Camphor & n.d. & n.d. & n.d. & n.d. & $0.005 \pm 0.00$ & $0.003 \pm 0.00$ \\
\hline 18 & 5-undecen-3-yne, (E)- & $1.2 \pm 0.30$ & n.d. & n.d. & n.d. & n.d. & n.d. \\
\hline 19 & Menthofuran & n.d. & n.d. & $0.001 \pm 0.00$ & $0.002 \pm 0.00$ & $0.002 \pm 0.00$ & $0.001 \pm 0.00$ \\
\hline 20 & Isomenthone & $0.002 \pm 0.00$ & $0.008 \pm 0.00$ & $0.001 \pm 0.00$ & n.d. & n.d. & n.d. \\
\hline 21 & Isomenthol & n.d. & n.d. & $0.01 \pm 0.00$ & n.d. & n.d. & $0.001 \pm 0.00$ \\
\hline 22 & Borneol & n.d. & $0.004 \pm 0.00$ & n.d. & n.d. & n.d. & n.d. \\
\hline 24 & Terpinen-4-ol & n.d. & $0.04 \pm 0.00$ & n.d. & n.d. & $0.005 \pm 0.00$ & $0.001 \pm 0.00$ \\
\hline 25 & $\alpha$-terpineol & n.d. & $0.05 \pm 0.01$ & $0.06 \pm 0.01$ & $0.01 \pm 0.00$ & n.d. & $0.002 \pm 0.00$ \\
\hline 26 & Estragole & $0.01 \pm 0.00$ & n.d. & n.d. & n.d. & n.d. & $0.3 \pm 0.02$ \\
\hline 27 & Verbenone & n.d. & n.d. & n.d. & n.d. & $0.003 \pm 0.00$ & $0.002 \pm 0.00$ \\
\hline 28 & Anethol & n.d. & n.d. & n.d. & n.d. & n.d. & $96.8 \pm 1.80$ \\
\hline 29 & $\beta$-caryophyllene & $0.4 \pm 0.02$ & $0.1 \pm 0.00$ & $0.1 \pm 0.01$ & n.d. & n.d. & n.d. \\
\hline 30 & Bergamottin & n.d. & $0.2 \pm 0.01$ & n.d. & n.d. & n.d. & n.d. \\
\hline 31 & $\alpha$-caryophyllene & $0.04 \pm 0.00$ & n.d. & n.d. & n.d. & n.d. & n.d. \\
\hline 32 & $\beta$-eudesmene & $9.8 \pm 0.20$ & n.d. & n.d. & n.d. & n.d. & n.d. \\
\hline 33 & Eremophilane & $1.2 \pm 0.04$ & n.d. & n.d. & n.d. & n.d. & n.d. \\
\hline 34 & Bisabolene & n.d. & $0.2 \pm 0.00$ & n.d. & n.d. & n.d. & n.d. \\
\hline 35 & Isoeugenol & $0.05 \pm 0.00$ & n.d. & $0.03 \pm 0.00$ & n.d. & n.d. & n.d. \\
\hline 36 & 1-(2,4-Dimethylphenyl)propan-1-one & $2.5 \pm 0.30$ & n.d. & n.d. & n.d. & n.d. & n.d. \\
\hline
\end{tabular}


Table 3. Cont

\begin{tabular}{|c|c|c|c|c|c|c|c|}
\hline & Compound & $\begin{array}{c}\text { Apium } \\
\text { graveolens }\end{array}$ & $\begin{array}{l}\text { Citrus } \\
\text { limon }\end{array}$ & $\begin{array}{c}\text { Citrus } \\
\text { paradisi }\end{array}$ & $\begin{array}{c}\text { Citrus } \\
\text { reticulata }\end{array}$ & $\begin{array}{c}\text { Citrus } \\
\text { sinensis }\end{array}$ & $\begin{array}{c}\text { Foeniculum } \\
\text { vulgare }\end{array}$ \\
\hline 37 & Allyl phenoxyacetate & $8.2 \pm 0.40$ & n.d. & n.d. & n.d. & n.d. & n.d. \\
\hline \multirow[t]{7}{*}{38} & 2-(2-propenyl)-furan & $1.9 \pm 0.20$ & n.d. & n.d. & n.d. & n.d. & n.d. \\
\hline & Oxygenated monoterpenes & n.d. & n.d. & n.d. & n.d. & n.d. & 0.4 \\
\hline & Alcohols & n.d. & 0.1 & 0.1 & n.d. & n.d. & n.d. \\
\hline & Phenylpropanoids & n.d. & n.d. & n.d. & n.d. & n.d. & 97.0 \\
\hline & Esthers & 8.2 & n.d. & n.d. & n.d. & n.d. & n.d. \\
\hline & Sesquiterpene hydrocarbons & 11.5 & 0.4 & 0.1 & n.d. & n.d. & n.d. \\
\hline & Others & 5.6 & 0.2 & n.d. & n.d. & n.d. & n.d. \\
\hline
\end{tabular}


Apium EO showed the limonene as main compound accounting more than $70 \%$ followed by $\beta$-eudesmene; similar composition was noticed by Zorga et al. [15]. Allyl phenoxyacetate was identified as a third compound; it was recently found in celery leaves EO by Stan et al. [16].

As expected, citrus samples reported limonene as the main compound. Its content ranged from 87 to $99 \%$. The lowest content was detected in citrus lemon sample; all the other ones showed a content higher than $99 \%$. $\beta$-pinene and $\gamma$-terpinene were the second and third compounds, respectively, in citrus lemon EO (6\%). Similar results were reported by Singh et al. [17]. All Citrus samples, except orange essential oil, did not comply with the ISO standards; this could be justified with the provenience of the raw material (Turkey) as reported by Singh et al. [17].

F. vulgare EO reported high amount of anethol that was about $97 \%$ of total compounds. Other compounds were limonene and $\alpha$-pinene; fenchone and estragole were also detected in small amounts. These results agreed with those reported by Ferioli and co-workers [18] in sweet fennel.

\subsection{Antioxidant Activity of the Essential Oils}

The antioxidant activity of the essential oils was evaluated by two different assays such as DPPH and ABTS. Table 4 shows the results of DPPH assay.

Table 4. DPPH Free Radical-Scavenging Capacity of different essential oils from plants and fruits.

\begin{tabular}{cc}
\hline Essential Oils & IC $_{\mathbf{5 0}}$ DPPH $(\mathbf{m g} / \mathbf{m L})$ \\
\hline Aromatic plants EOs & \\
\hline Cinnamomum cassia & 0.05 \\
Cinnamomum zeylanicum & 0.01 \\
Mentha piperita & 22.98 \\
Origanum vulgare & 0.29 \\
Rosmarinus officinalis & 11.19 \\
Salvia lavandulifolia & 41.82 \\
Thymus vulgaris & 0.31 \\
\hline Fruit and vegetables EOs & \\
\hline Apium graveolens & 25.89 \\
Citrus limon & 49.06 \\
Citrus paradisi & 87.67 \\
Citrus reticulata & 38.79 \\
Citrus sinensis & 47.30 \\
Foeniculum vulgare & 105.32 \\
\hline Ascorbic acid & 0.003 \\
\hline
\end{tabular}

Aromatic plants EOs. Cinnamon EOs have demonstrated to have the strongest antioxidant activity between the others and the minor differences with ascorbic acid. From them, C. zeylanicum leaf EO is slightly better with an $\mathrm{IC}_{50}$ five times lower than C. cassia bark EO.

Comparing with other authors, this C. zeylanicum leaf EO has higher free radicalscavenging than the EOs from Cinnamomum leaves as C. tamala $\left(\mathrm{IC}_{50}: 1.65 \mathrm{mg} / \mathrm{mL}\right)$ [19], C. griffithii (IC $\mathrm{IC}_{50}: 0.082 \mathrm{mg} / \mathrm{mL}$ ), C. macrocarpum ( $\mathrm{IC}_{50}: 0.099 \mathrm{mg} / \mathrm{mL}$ ) [20], and C. malabathrum $\left(\mathrm{IC}_{50}: 1.7 \mathrm{mg} / \mathrm{mL}\right)$ [21]. These differences are due to their content in eugenol, as they only reached EOs with a content between 38.5-52\% [19,20], while ours had $95.2 \%$ (Table 4). The same happens with the EO reported by Srirmavaratharajan et al. [22] from C. wightii leaves that contain $72.6-85.9 \%$ of benzyl benzoate as major compound, causing them to have an $\mathrm{IC}_{50}$ of $3.49 \mathrm{mg} / \mathrm{mL}$, much higher than the reported in this work. Moreover, it has 23 times higher antioxidant activity than other commercial EO from the same Cinnamomum specie leaves $\left(\mathrm{IC}_{50}: 0.23 \mathrm{mg} / \mathrm{mL}\right)$ that had $48.8 \%$ of eugenol [23]. Therefore, the antioxidant activity of Cinamomum leaf EO can be attributed to the content in eugenol. 
C. cassia bark $\mathrm{EO}$ has reported similar $\mathrm{IC}_{50}$ to other authors in other varieties of Cinnamomum bark EOs, such as C. altissium $\left(\mathrm{IC}_{50}: 0.04 \mathrm{mg} / \mathrm{mL}\right.$ ) [24] and C. griffithii $\left(\mathrm{IC}_{50}\right.$ : $0.07 \mathrm{mg} / \mathrm{mL}$ ) [20], and lower than others $\left(\mathrm{IC}_{50}: 0.10 \mathrm{mg} / \mathrm{mL}\right)[20,23]$, although in all cases the major compounds are different. It is remarkable that they did not name the compound cinnamaldehyde, which is the major compound reported by several studies [25], and it is in concordance with this work in which it has been found in an amount of $91.9 \%$.

Following them, O. vulgare flower/leaf and T. vulgaris EOs have also demonstrated high antioxidant activity compared with the others, occupying the third and fourth positions, respectively. The $\mathrm{IC}_{50}$ obtained for the $\mathrm{O}$. vulgare $\mathrm{EO}$ with major compound carvacrol $(84.5 \%)$ is in concordance with other authors in EOs obtained by hydrodistillation as Hamada et al. [26] with an $\mathrm{IC}_{50}: 0.30 \mathrm{mg} / \mathrm{mL}$ ( $48.4 \%$ of carvacrol), and Sokmen et al. [27] who reported $\mathrm{IC}_{50}: 0.31 \mathrm{mg} / \mathrm{mL}$ (64.3\% of carvacrol). Boskovic et al. [28] also agreed with us, reporting an $\mathrm{IC}_{50}$ of $0.33 \mathrm{mg} / \mathrm{mL}$ for the O. vulgare EO obtained from a Serbian company.

T. vulgaris $\mathrm{EO}$ has demonstrated slightly lower antioxidant activity than those obtained by hydrodistillation $\left(\mathrm{IC}_{50}: 0.159-0.243 \mathrm{mg} / \mathrm{mL}\right.$ ) $[29,30]$ maybe as the differences in composition. They reported thymol as major compound at concentrations of $36.5-55.3 \%$ followed by carvacrol $28.7-29.8 \%$ and $p$-cymene $10-11.2 \%$. In this case, we have found thymol $61.2 \%$, $p$-cymene $16.7 \%$, and carvacrol $2.6 \%$, so the difference can be attributed to the reduced content in carvacrol compared with them. However, it is in the range of values reported by Boskovic et al. [28] ( $\mathrm{IC}_{50}: 0.48 \mathrm{mg} / \mathrm{mL}$ ) and Aazza et al. [31] ( $\mathrm{IC}_{50}$ : $0.26 \mathrm{mg} / \mathrm{mL}$ ) in commercial ones from Serbia and Morocco, respectively.

After those four, the EOs with higher antioxidant activity are from $R$. officinalis leaf and M. piperita leaf. R. officinalis leaf $\mathrm{EO}$ has demonstrated less effectivity than those obtained by other authors that have reported $\mathrm{IC}_{50}$ from 0.52 to $3.48 \mathrm{mg} / \mathrm{mL}$ [32-36]. However, in contrast, the scavenging activity is better than the reported by Risaliti et al. [37] who obtained $25 \%$ of inhibition with $4.23 \mathrm{mg} / \mathrm{mL}$ of $R$. officinalis EO from a Greek company (our EO would need $3.77 \mathrm{mg} / \mathrm{mL}$ for this $25 \%$ of inhibition). They found lower content in $\beta$-myrcene ( $0.9 \%$ in front of $30.75 \%)$, camphor $(11.7 \%$ in front of $20.7 \%)$, and $\beta$-pinene $(8.3 \%$ in front of $11.9 \%)$, despite the fact that its content in eucalyptol is higher $(48.7 \%$ in front of $14.8 \%$ ). This seems to indicate that the minor compounds also contribute to the reducing power.

The antioxidant activity shown by M. piperita $\mathrm{EO}$ is in concordance with that reported by Wu et al. [38] ( $\mathrm{IC}_{50}: 22.77 \mathrm{mg} / \mathrm{mL}$ ) in an USA commercial sample, and higher than that reported by Fatemi et al. [39] $\left(\mathrm{IC}_{50}: 25.80 \mathrm{mg} / \mathrm{mL}\right)$ in an $\mathrm{EO}$ obtained by hydro-distillation, and Stanojevic et al. [40] ( $\left.\mathrm{IC}_{50}: 58.41 \mathrm{mg} / \mathrm{mL}\right)$ in a EO from a Serbian company. All of them had very similar composition being the major compounds menthol (38.4-52.4\%), menthone (13.8-24.9\%), menthyl acetate (3.9-6.5\%), and eucalyptol (3.9-5.6\%), which is in concordance with us (menthol $39.7 \%$, menthone $29.8 \%$, menthyl acetate $6.1 \%$, and eucalyptol $4.9 \%)$.

Following them is S. lavandulifolia EO. It has demonstrated higher $\mathrm{IC}_{50}$ than other S. lavandulifolia leaves EO obtained by hydro-distillation ( $\left.\mathrm{IC}_{50}: 0.97-8.31 \mathrm{mg} / \mathrm{mL}\right)$ [41,42]. This lower antioxidant activity can be attributed to the inversion in amount in the major compounds, namely camphor (20.3-33.6\%) > eucalyptol (15.0-22.2\%) $>\alpha$-thujene (14.9$21.4 \%$ ) in front of eucalyptol $>$ camphor $>$ camphene that are found in our S. lavandulifolia EO. Risaliti et al. [37] who used Salvia triloba leaves EO from a Greek company achieved a $25 \%$ of inhibition with $4.47 \mathrm{mg} / \mathrm{mL}$ and ours could achieve that with $14.5 \mathrm{mg} / \mathrm{mL}$, although the compositions are very similar (S. triloba eucalyptol $(46.68 \%)>$ camphor $(10.5 \%)>\alpha$-pinene $(8.5 \%)>$ camphene $(6.7 \%)>\beta$-pinene $(6.7 \%)$, and S. lavandulifolia eucalyptol $(38.6 \%)>$ camphor $(23.6 \%)>$ camphene $(7.7 \%)>\alpha$-pinene $(5.5 \%)>\beta$-pinene $(5.1 \%))$. However, it seems to have better antioxidant activity than other EOs from other varieties, such as Salvia kiangsiensis of which, according with Fang et al. [43], $10 \mathrm{mg} / \mathrm{mL}$ are needed to have $4.3 \%$ of inhibition, meanwhile with ours only $1.5 \mathrm{mg} / \mathrm{mL}$ would be necessary, and the compositions are totally different. 
Fruit and vegetables EOs. A. graveolens seed $\mathrm{EO}$ is the vegetable EO that has shown higher antioxidant activity. However, its activity is lower than that reported by Hassanen et al. [44] who, with $0.9 \mathrm{mg} / \mathrm{mL}$ of $A$. graveolens seed EO hydro-distillated, achieved $74.3 \%$ of inhibition. With this concentration of our EO, we could reach only $35 \%$ of inhibition. Although the compositions are similar, they obtained an EO with higher amount of $\beta$-selinene $(27.0 \%$ in front of $9.8 \%$ ) which could be the responsible of this increment in the antioxidant activity.

Taking into account the citrus EOs, the order according with their antioxidant strength is C. reticulata $>$ C. sinensis $>C$. paradisii. They have very similar composition with Dlimonene as major compound counting for $99 \%$ in all cases. However, the difference in the reducing power between them could be due to its content in the second major compound $\beta$-myrcene that counts for $0.40,0.36$, and $0.31 \%$, respectively. Comparing with Kamal et al. [45], they obtained 24.1, 18.5, and $14.0 \%$ of inhibition with $0.1 \mathrm{mg} / \mathrm{mL}$ of C. reticulata, C. sinensis, and C. paradisii EOs, respectively, which corroborate the order and differences between them found in this study. The values of $\mathrm{IC}_{50}$ obtained for $C$. sinensis and C. paradisii EOs are in the range of the values reported by Phi et al. [46] (28.5-63.43 and $45.7-86.3 \mathrm{mg} / \mathrm{mL}$, respectively). If comparing our $C$. sinensis peel EO with C. sinensis leaves $\mathrm{EO}$, the studies revealed that those that come from leaves have much higher antioxidant activities with $\mathrm{IC}_{50}$ between $0.75-1.49 \mathrm{mg} / \mathrm{mL}$ [47]. This is mainly attributed to the composition that consists of $16.9 \% \beta$-pinene, $13.8 \%$ D-limonene, and $7.5 \%$ of $\beta$-ocimene as major compounds. In this group of essential oils, it can be appreciated that the antioxidant activity can clearly be attributed to the limonene content, but also to the content in other monoterpenoids. For the $C$. paradisii peel EO, although the composition reported by Kaanin-Boudraa et al. [48] and Ou et al. [49] are very similar to that reported here, they obtained an $\mathrm{IC}_{50}$ of $40 \mathrm{mg} / \mathrm{mL}$, amounting to half of our total. Another Citrus EO, C. limon peel EO, has been evaluated apart from the rest due to its different composition found (Table 3). Compared with the others, it has lower D-limonene (87.0\%), $\beta$-pinene $(6.0 \%)$, and $\gamma$-terpineno. Ben Miri et al. [50] found that C. sinensis EO had the double antioxidant activity than C. limon, while in this study, both EOs have demonstrated similar $\mathrm{IC}_{50}$. However, the antioxidant activity found is in concordance with Guo et al. [51], who reported $32.8 \%$ of inhibition with $30 \mathrm{mg} / \mathrm{mL}$. Moreover, it has been demonstrated that C. limon peel $\mathrm{EO}$ has lower antioxidant activity than $\mathrm{C}$. limon leaves $\mathrm{EO}$ according with Fancello et al. [52] who reported an $\mathrm{IC}_{50}$ of $11.9 \mathrm{mg} / \mathrm{mL}$.

The EO which has shown least antioxidant activity between all tested is F. vulgare var. dulce EO. The result obtained is very far from those reported by Kalleli et al. [53] from Tunisian $\left(\mathrm{IC}_{50}: 0.20-0.49 \mathrm{mg} / \mathrm{mL}\right)$ and French $\left(\mathrm{IC}_{50}: 0.59-0.63 \mathrm{mg} / \mathrm{mL}\right)$ F. vulgare seeds EOs. However, it is closer to the values reported by Ahmed et al. [54] for Chinese samples $\left(\mathrm{IC}_{50}: 15.66 \mathrm{mg} / \mathrm{mL}\right)$ and totally in concordance with the Egyptian ones $\left(\mathrm{IC}_{50}\right.$ : $141.82 \mathrm{mg} / \mathrm{mL}$ ). According to them, the Tunisian and Chinese ones agree with us about the major compound, anethole (54.3-78.3\%) but they revealed higher amounts of estragole (17.1-20.2\%), L-fenchone (7.4-12.1\%), and D-limonene (2.4-4.7\%). Otherwise, the French one had as major compound estragole (44.7-88.9\%), with lower amounts of anethole (14.0-36.3\%). These compositional differences could be responsible of the changes in the antioxidant activity.

Correlation DPPH vs. ABTS. All the exposed data obtained by the DPPH technique is in concordance with the results obtained with the ABTS technique. As shown in Figure 1, there was significant correlation between them $\left(\mathrm{r}=0.9291, \mathrm{r}^{2}=0.8681\right.$, and $\left.p<0.001\right)$, which indicated that both techniques could be used to investigate the antioxidant activity of plants and fruit and vegetables EOs although they have not been found enough references to compare. 


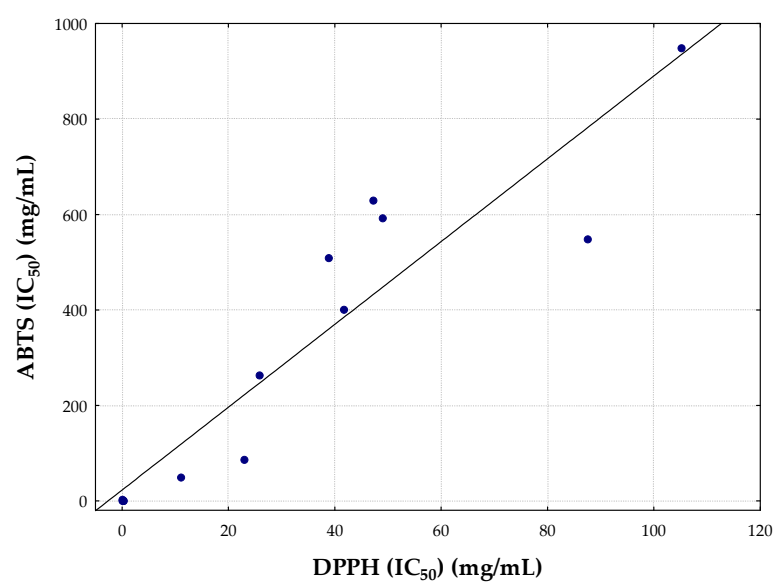

Figure 1. Correlation between the $\mathrm{IC}_{50}$ values obtained by DPPH and ABTS assays in aromatic plants and fruits and vegetables EOs.

\subsection{Antimicrobial Activity against Bacteria and C. albicans}

Of 13 essential oils tested, 11 showed inhibitory activity against one or more bacteria or C. albicans (Table 5). Those essential oils that showed diameters of the zones of inhibition higher than or equal to $28 \mathrm{~mm}$ ( $8 \mathrm{~mm}$ well-diameter included) were considered to have a strong inhibitory effect, between 16 and $28 \mathrm{~mm}$ as moderately active inhibitors, between 12 and $16 \mathrm{~mm}$ as mild inhibitors, and less than $12 \mathrm{~mm}$ as no or low inhibitors [55]. Thus, Eos obtained from C. cassia, T. vulgaris, and O. vulgare proved to be strong inhibitors against most of the bacterial species tested, both Gram-negative and Gram-positive, and the yeast C. albicans.

Table 5. Measure of inhibition zone diameters $(\mathrm{mm})$ for essential oils against bacterial strains.

\begin{tabular}{|c|c|c|c|c|c|c|}
\hline Essential Oils & S. aureus & MRSA & E. coli & S. Typhimurium & L. monocytogenes & C. albicans \\
\hline Thymus vulgaris & $33.0 \pm 1.0$ & $33.0 \pm 2.0$ & $38.0 \pm 3.6$ & $41.0 \pm 1.0$ & $35.3 \pm 5.0$ & $58.0 \pm 2.6$ \\
\hline Origanum vulgare & $28.7 \pm 5.5$ & $30.7 \pm 3.8$ & $33.3 \pm 4.2$ & $35.7 \pm 1.2$ & $31.7 \pm 2.9$ & $50.7 \pm 1.2$ \\
\hline Rosmarinus officinalis & $10.7 \pm 1.2$ & $10.0 \pm 0.0$ & $11.0 \pm 1.7$ & $12.7 \pm 1.2$ & $\mathrm{~N}$ & $18.7 \pm 2.3$ \\
\hline Apium graveolens & $12.0 \pm 2.6$ & $11.3 \pm 0.6$ & $\mathrm{~N}$ & $13.0 \pm 1.0$ & $11.7 \pm 1.5$ & $20.7 \pm 1.2$ \\
\hline Salvia lavandulifolia & $10.3 \pm 0.6$ & $10.3 \pm 0.6$ & $10.7 \pm 0.6$ & $13.3 \pm 0.6$ & $\mathrm{~N}$ & $25.0 \pm 3.0$ \\
\hline Cinnamomum zeylanicum & $18.3 \pm 2.3$ & $18.3 \pm 1.2$ & $21.3 \pm 1.2$ & $21.7 \pm 1.2$ & $14.3 \pm 0.6$ & $34.7 \pm 0.6$ \\
\hline Cinnamomum cassia & $36.0 \pm 3.5$ & $34.7 \pm 2.3$ & $29.0 \pm 4.6$ & $28.0 \pm 1.7$ & $28.0 \pm 2.0$ & $55.7 \pm 3.5$ \\
\hline Citrus sinensis & $\mathrm{N}$ & $\mathrm{N}$ & $\mathrm{N}$ & $\mathrm{N}$ & $\mathrm{N}$ & $\mathrm{N}$ \\
\hline Citrus reticulata & $10.3 \pm 0.6$ & $10.0 \pm 0.0$ & $\mathrm{~N}$ & $14.0 \pm 1.0$ & $10.3 \pm 0.6$ & $22.0 \pm 1.7$ \\
\hline Citrus limon & $\mathrm{N}$ & $\mathrm{N}$ & $\mathrm{N}$ & $\mathrm{N}$ & $\mathrm{N}$ & $\mathrm{N}$ \\
\hline Citrus paradise & $\mathrm{N}$ & $\mathrm{N}$ & $\mathrm{N}$ & $\mathrm{N}$ & $\mathrm{N}$ & $\mathrm{N}$ \\
\hline Foeniculum vulgare & $\mathrm{N}$ & $\mathrm{N}$ & $\mathrm{N}$ & $12.0 \pm 2.0$ & $\mathrm{~N}$ & $12.7 \pm 2.5$ \\
\hline Mentha piperita & $11.0 \pm 0.0$ & $11.0 \pm 1.0$ & $13.3 \pm 1.5$ & $22.3 \pm 2.5$ & $\mathrm{~N}$ & $40.3 \pm 4.0$ \\
\hline $\mathrm{AA}$ & $15.3 \pm 0.6$ & $17.3 \pm 0.6$ & $32.3 \pm 4.0$ & $30.7 \pm 1.2$ & $19.0 \pm 1.0$ & $25.7 \pm 1.2$ \\
\hline
\end{tabular}

Results are presented by mean values from three experiments \pm standard deviations. S. aureus: Staphylococcus aureus; MRSA: methicillinresistant Staphylococcus aureus; E. coli: Escherichia coli; S. Typhimurium: Salmonella enterica serovar Typhimurium; L. monocytogenes: Listeria monocytogenes; C. albicans: Candida albicans; N: no inhibition zone; AA: antimicrobial agents (ciprofloxacin $2 \mathrm{mg} / \mathrm{mL}$ for MRSA, $0.1 \mathrm{mg} / \mathrm{mL}$ for L. monocytogenes, $0.01 \mathrm{mg} / \mathrm{mL}$ for the rest of bacteria, and ketoconazole $0.01 \mathrm{mg} / \mathrm{mL}$ for C. albicans).

Cassia bark essential oil was the most effective oil against all the strains tested showing zones of inhibition that ranged between 28.0 and $55.7 \mathrm{~mm}$ of diameters and higher than inhibition zones of the antimicrobial agents. Previous research is consistent with our results as essential oils of $C$. cassia have shown potent antimicrobial activity against $L$. monocytogenes, E.coli, S. Typhimurium [56], Candida glabrata, and C. albicans [57]. Firmino et al. [58] showed that essential oils extracted from cassia bark, as well as its main component, trans-cinnamaldehyde, in a concentration range of 0.25 to $0.50 \mathrm{mg} / \mathrm{mL}$, inhibited the growth of the planktonic forms of S. aureus and E. coli, and reduced biomass in biofilms of both bacteria by more than $99.9 \%$. Trans-Cinnamaldehyde is an unsaturated aldehyde 
that possesses an acrolein group ( $\alpha, \beta$ - unsaturated carbonyl moiety) which is essential for antimicrobial activity [59]. Trans-Cinnamaldehyde has been shown to possess substantial antimicrobial activity against Gram-positive and Gram-negative bacteria, including L. monocytogenes, S. aureus, S. Typhimurium, E. coli, and Pseudomonas aeruginosa [56,60-62]. At sublethal concentrations, this compound is capable of inhibiting cell division by acting on the FtsZ protein, but at higher concentrations, it has a bactericidal action as it affects the integrity of bacterial membranes [63]. The antimicrobial activity of the essential oils of cassia observed in this work is attributable to trans-cinnamaldehyde, which represented $91.9 \%$ of the extract.

Of particular interest is the strong antibacterial activity of the essential oils of thyme and oregano EOs, herbs used frequently in gastronomy, against all the bacterial strains tested, as most of them have been implicated as causal agents of foodborne disease outbreaks and food quality degradation $[64,65]$. This strong antimicrobial activity is in concordance with other studies. Silva et al. [66] reported that thyme and oregano EOs showed significant antibacterial activity against L. monocytogenes, S. Typhimurium, S. aureus, and E. coli, and Bozin et al. [67] found a strong antibacterial activity of oregano and thyme EOs, even on multiresistant strains of E. coli, S. Typhimurium, and S. aureus. Our results showed inhibition diameters similar or higher than those of the ciprofloxacin against the tested bacterial strains, and higher than those of ketoconazole diameters against C. albicans.

The efficacy of these EOs can be attributed to the activity of phenolic compounds carvacrol and thymol, the major compounds of oregano and thyme essential oils, respectively. In this work, oregano EO was mainly composed of carvacrol (84.52\%) and thymol (1.62\%), whereas thyme oil contained $61.21 \%$ of thymol and $2.58 \%$ of carvacrol. Carvacrol has been reported to be active against C. albicans, L. monocytogenes, E. coli, S. Typhimurium, S. aureus, Shigella sonnei, and Shigella flexneri [56,68-71] and thymol has shown activity against E. coli, S. Typhimurium, S. aureus, L. monocytogenes, S. sonnei, S. flexneri, and Bacillus cereus [56,70-72]. Thymol is structurally analogous to carvacrol, but have a free hydroxyl group, the radical essential for antimicrobial activity [69], at a different location on the phenolic ring. Both compounds interact with the cell membrane, making it permeable due to the introduction of lipophilic group into the ordered structure of the lipid bilayer $[63,73]$.

The essential oil obtained from C. zeylanicum leaf showed moderate inhibition against bacterial strains with inhibition zones between 14.3 and $21.7 \mathrm{~mm}$, and strong inhibition for C. albicans. Regarding antimicrobial agents, this essential oil showed inhibition zones similar to ciprofloxacin against bacterial strains, but higher than those of ketoconazole against C. albicans. In agreement with our results, Ebani et al. [74], Prabuseenivasan et al. [75], and Brnawi et al. [76] reported antibacterial activity of cinnamon oil against several Grampositive and Gram-negative bacteria. The antimicrobial activity of cinnamon oil can be ascribed to eugenol, a phenylpropene that was found in this work at $95.23 \%$. This compound alters the membrane and the transport of ions and ATP and modifies the fatty acid profile [63]. Eugenol is active against foodborne pathogens such as E. coli, L. monocytogenes, S. aureus, S. Typhimurium, Bacillus subtilis, and B. cereus $[69,77,78]$.

On the contrary to most of the EOs, essential oils obtained from citric fruits exhibited low or no inhibition against all tested bacteria, except the mandarin essential oil that mildly inhibited S. Typhimurium with inhibition zones about $14 \mathrm{~mm}$ and moderately to C. albicans with inhibition zones of $22 \mathrm{~mm}$. D-limonene is one of the major compounds of the citrus essential oils, a monoterpene whose antimicrobial activity depends on the alkyl group [63]. In this work, it was found as a major compound in lemon, mandarin, sweet orange, and pink grapefruit EOs (87.0, 99.0, 99.2, and 99.0, respectively). In addition to limonene, $\beta$-pinene, and $\gamma$-terpinene, biological precursors of phenolic compounds, were found in lemon at 6.0 y 3.8\%, respectively. Some terpenes do not possess high antimicrobial activity when they are used as a single compound. Such is the case of $p$-cymene, one of the most important components of thyme essential oil, which did not show antimicrobial activity against E. coli, S. sonnei, and S. flexneri using the agar well diffusion assay [71]. Similarly, 21 terpenoids such as limonene, $\alpha$-pinene, $\beta$-pinene, $\gamma$-terpinene $\delta$ - 3 -carene, $(+)$-sabinene, 
and $\alpha$-terpinene showed low inhibition of the bacterial growth, whereas all essential oils exhibited considerable inhibitory effects against 25 different genera of bacteria, including plant pathogens, food poisoning, and spoilage bacteria [79], suggesting that a combination of bioactive compounds in a suitable proportion, is needed to achieve a high and effective overall activity.

The minimal inhibitory concentration (MIC) of essential oils against bacteria and C. albicans was determined by the agar dilution method and was expressed in $\mathrm{mg} / \mathrm{mL}$ (Table 6). MIC values obtained are consistent with previous diameters of inhibition zones. The essential oil obtained from C. cassia bark showed the lowest MIC values between 0.14 and $0.28 \mathrm{mg} / \mathrm{mL}$ for bacteria, and $<0.14 \mathrm{mg} / \mathrm{mL}$ for C. albicans. Similarly, cinnamon, thyme and oregano essential oils showed great MIC values for all tested strains showing MIC values between 0.28 and $2.25 \mathrm{mg} / \mathrm{mL}$. A moderate effect was observed with the rosemary, celery, sage, and fennel EOs that showed MIC values between 1.125 and $4.5 \mathrm{mg} / \mathrm{mL}$, and the citric fruits essential oils were the less effective EOs with MIC values from 18 to $72 \mathrm{mg} / \mathrm{mL}$ for bacteria, and between 4.5 and $18 \mathrm{mg} / \mathrm{mL}$ for C. albicans.

Table 6. Minimum Inhibitory Concentration MIC $(\mathrm{mg} / \mathrm{mL})$ for essential oils against bacterial strains.

\begin{tabular}{|c|c|c|c|c|c|c|}
\hline Essential Oils & S. aureus & MRSA & E. coli & S. Typhimurium & L. monocytogenes & C. albicans \\
\hline Thymus vulgaris & 2.25 & 1.125 & 1.125 & 1.125 & 1.125 & 0.56 \\
\hline Origanum vulgare & 1.125 & 1.125 & 1.125 & 0.56 & 1.125 & 0.56 \\
\hline Rosmarinus officinalis & 36 & 36 & 18 & 9 & 18 & 4.5 \\
\hline Apium graveolens & 4.5 & 4.5 & 36 & 9 & 4.5 & 1.125 \\
\hline Salvia lavandulifolia & 9 & 9 & 9 & 4.5 & 4.5 & 2.25 \\
\hline Cinnamomum zeylanicum & 2.25 & 1.125 & 1.125 & 1.125 & 2.25 & 0.28 \\
\hline Cinnamomum cassia & 0.28 & 0.28 & 0.28 & 0.28 & 0.14 & $<0.14$ \\
\hline Citrus sinensis & 72 & 72 & 36 & 36 & 36 & 18 \\
\hline Citrus reticulata & 36 & 36 & 36 & 36 & 18 & 4.5 \\
\hline Citrus limon & 72 & 72 & 72 & 36 & 36 & 18 \\
\hline Citrus paradise & 72 & 72 & 72 & 36 & 72 & 9 \\
\hline Foeniculum vulgare & 36 & 72 & 36 & 4.5 & 36 & 2.25 \\
\hline Mentha piperita & 4.5 & 2.25 & 2.25 & 1.125 & 4.5 & 1.125 \\
\hline
\end{tabular}

S. aureus: Staphylococcus aureus; MRSA: methicillin-resistant Staphylococcus aureus; E. coli: Escherichia coli; S. Typhimurium: Salmonella enterica serovar Typhimurium; L. monocytogenes: Listeria monocytogenes; and C. albicans: Candida albicans.

\subsection{Antifungal Activity of Essential Oils against B. cinerea}

Antifungal properties of the thirteen essential oils were assessed against $B$. cinerea by agar well-diffusion and agar dilution methods. The percentage of inhibition of mycelial growth and the MIC values were determined after 7 days of incubation (Table 7). Among all the essential oils tested, oregano and thyme EOs showed the highest inhibition (98.5 and $98.2 \%$, respectively), followed by fennel and mint EOs that inhibited 93.8 and $93.1 \%$ of the mycelia growth, respectively. Mild inhibition was observed by celery EO $(48.8 \%)$ and low or no inhibition by EOs obtained from citric fruits (3.4 to 18.4\%). Antimicrobial control with ketoconazole inhibited completely the mycelial growth. The minimal inhibitory concentration values ranged from 0.07 to $9 \mathrm{mg} / \mathrm{mL}$. Although thyme and oregano EOs showed the highest mycelial reduction, cassia EO showed the lowest MIC value $(0.14 \mathrm{mg} / \mathrm{mL})$ followed by mint, oregano, fennel, thyme, cinnamon, and sage EOs that inhibited the fungus by concentrations of 0.56 to $2.25 \mathrm{mg} / \mathrm{mL}$. Rosemary, celery, orange, lemon, mandarin, and pink grapefruit EOs showed MIC values between 4.5 and $9 \mathrm{mg} / \mathrm{mL}$.

Previous research is in concordance with our results. The cassia and oregano EOs completely inhibited the mycelial growth of $B$. cinerea at $0.5 \mathrm{mg} / \mathrm{mL}$, and concentrations of $250 \mathrm{mg} / \mathrm{mL}$ thymol and $300 \mathrm{mg} / \mathrm{mL}$ carvacrol inhibited completely its spore germination [80]. Additionally, the growth of B. cinerea was completely inhibited by cassia [81], oregano, and rosemary essential oils [82]. Other research reported the antifungal activity of carvacrol, the main compound of oregano EO, and eugenol, the main compound of cinnamon EO, against B. cinerea [69]. At 500 ppm, cinnamon EOs completely inhibited the 
growth of $B$. cinerea from $72 \mathrm{~h}$ of contact with the EOs, whereas thyme EO achieved the same from $120 \mathrm{~h} \mathrm{[83].} \mathrm{According} \mathrm{to} \mathrm{our} \mathrm{results,} \mathrm{Palfi} \mathrm{et} \mathrm{al.} \mathrm{[84]} \mathrm{reported} \mathrm{inhibition} \mathrm{of} \mathrm{the}$ mycelial growth of $B$. cinerea in the presence of thyme, fennel, peppermint, rosemary, and sage essential oils, whereas lemon oil lacked inhibitory activity.

Table 7. Percentage of mycelium inhibition of B. cinerea and minimal inhibitory concentration (MIC) of essential oils.

\begin{tabular}{ccc}
\hline Essential Oils & Mycelium Inhibition (\%) & MIC (mg/mL) \\
\hline Thymus vulgaris & 98.2 & 1.125 \\
Origanum vulgare & 98.5 & 0.56 \\
Rosmarinus officinalis & 79.0 & 9 \\
Apium graveolens & 48.8 & 9 \\
Salvia lavandulifolia & 79.8 & 2.25 \\
Cinnamomum zeylanicum & 70.4 & 1.125 \\
Cinnamomum cassia & 81.5 & 0.14 \\
Citrus sinensis & 3.4 & 9 \\
Citrus reticulata & 3.6 & 9 \\
Citrus limon & 9.7 & 4.5 \\
Citrus paradise & 18.4 & 9 \\
Foeniculum vulgare & 93.1 & 1.125 \\
Mentha piperita & 93.8 & 0.56 \\
\hline
\end{tabular}

In addition to the dose-dependent effect observed in the MIC assay, some EOs inhibited the fungus by more than $90 \%$ at concentrations much lower than their minimum inhibitory concentration. Such is the case of the fennel EO that inhibited $95 \%$ of the fungus at a concentration as low as $0.07 \mathrm{mg} / \mathrm{mL}$ and thyme $\mathrm{EO}$ that inhibited the mycelia growth in $97.6 \%$ at $0.14 \mathrm{mg} / \mathrm{mL}$, a concentration 8 times lower than its MIC value (Figure 2). Similarly, only $0.56 \mathrm{mg} / \mathrm{mL}$ of the celery EO inhibited $90.2 \%$ of mycelia growth, 16 times lower than its MIC value. This result is comparable with those of other researchers, such as the case of Grul'ová et al. [85] that reported complete inhibition of B. cinerea with $500 \mathrm{ppm}$ oregano EO, and more than $80 \%$ with 100 ppm of oil incorporated into Potato Dextrose Agar.
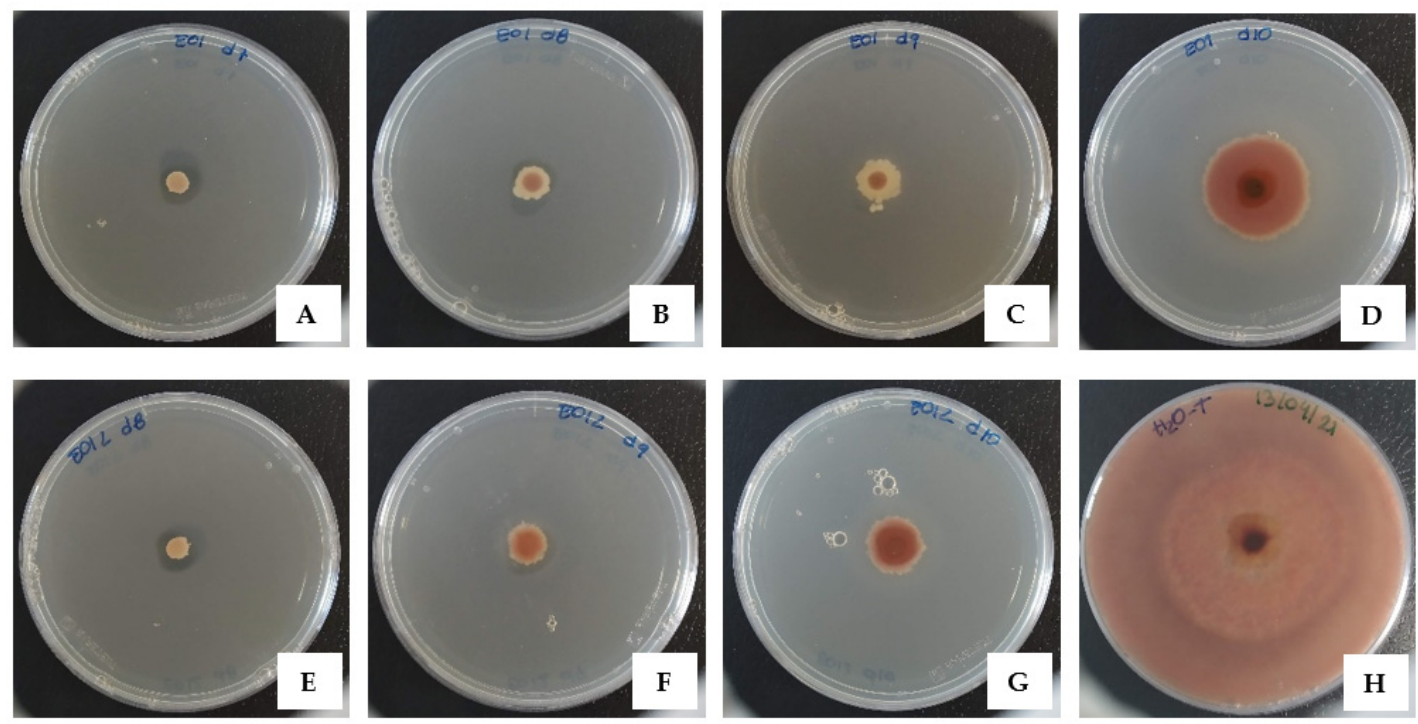

Figure 2. Inhibition of $B$. cinerea mycelium growth by thyme $\mathrm{EO}$ at $0.56 \mathrm{mg} / \mathrm{mL}(\mathbf{A}), 0.28 \mathrm{mg} / \mathrm{mL}(\mathbf{B}), 0.14 \mathrm{mg} / \mathrm{mL}$ (C), $0.07 \mathrm{mg} / \mathrm{mL}(\mathbf{D})$; and fennel EO at $0.28 \mathrm{mg} / \mathrm{mL}(\mathbf{E}), 0.14 \mathrm{mg} / \mathrm{mL}$ (F), and $0.07 \mathrm{mg} / \mathrm{mL}(\mathbf{G})$. Control of mycelium growth (H) after 7 days of incubation at $25^{\circ} \mathrm{C}$. 
B. cinerea is a phytopathogen that causes the grey mould disease in more than $200 \mathrm{crop}$ species worldwide such as grapes, cucumbers, tomatoes, strawberries, and leading to vast economic losses due to the severe damage in pre-and post-harvest [86,87]. Control strategies are carried out including chemical control, resistance induction and biological control. Benzimidazoles, dicarboximides, phenylpyrroles, aromatic hydrocarbons, and phenylcarbamates are the main chemical fungicides used to control it [88]. In addition to the toxicological risk presented by their residues, $B$. cinerea has developed resistance to most of these substances [89-91]. Essential oils have a significant interest as an alternative to chemical treatments as they are bio-sourced products and therefore more ecological [92].

Essential oils can act on fungus via inhibition of sporulation or producing cell damage [93]. Hydrophobic character enables EOs to break through lipids of cell membranes and mitochondria increasing fungal membranes permeability [92]. The changes in the fluidity may leak electrolytes or cellular contents resulting in protein metabolism alteration and calcium ion concentration [93]. Moreover, permeabilization of out and inner mitochondrial membrane leads to cell death by apoptosis and necrosis [94].

Although most of the antimicrobial activities of essential oils have been attributed to their major components, the total antimicrobial effect is the result of the synergism of all their components [95]. Therefore, the antimicrobial activity is not related to a single mechanism of action, as the essential oils have different bioactive compounds, and each one of them has different structural groups in their composition [96].

\section{Conclusions}

EOs from herbs and spices showed the highest antioxidant activity. Essential oils obtained from aromatic plants showed higher antibacterial and antifungal activity than those obtained from citric fruits. The most effective essential oils were those of $C$. cassia, T. vulgaris, and O. vulgare. Although fennel essential oil reported the lowest antioxidant activity, it showed very good antimicrobial activity against $B$. cinerea, one of the postharvest pathogen microorganisms in fruits and vegetables, thus it could be considered in active packaging production or food coating. The strong antimicrobial activity of essential oils and the broad spectrum they showed provide evidence that they may be used for prolonging the shelf life of food products, developing functional foods, and for protecting plants and crops.

Supplementary Materials: The following are available online at https://www.mdpi.com/article/10 .3390 /biology10111091/s1, Figure S1: GS-MS chromatograms of fruit and vegetables, and aromatic herbs and spices essential oils.

Author Contributions: Conceptualization, V.V. and A.M.G.-C.; formal analysis, M.d.C.R.-D., S.D.-M.-P. and V.V.; investigation, M.d.C.R.-D., S.D.-M.-P. and V.V.; data curation, E.J.G.-H., M.J.-V. and A.R.-B.; writing—original draft preparation, M.d.C.R.-D., S.D.-M.-P. and V.V.; writing—review and editing, M.J.-V., A.R.-B., B.G.-V. and V.V.; supervision, V.V.; and funding acquisition, V.V. ands A.M.G.-C. All authors have read and agreed to the published version of the manuscript.

Funding: This study is part of the project SHEALTHY that has received funding from European Union's Horizon 2020 research and innovation programme under grant agreement No 817936.

Institutional Review Board Statement: Not applicable.

Informed Consent Statement: Not applicable.

Data Availability Statement: Not applicable.

Acknowledgments: Vito Verardo thanks the Spanish Ministry of Economy and Competitiveness (MINECO) for "Ramon y Cajal" contract (RYC-2015-18795).

Conflicts of Interest: The authors declare no conflict of interest. 


\section{References}

1. Maurya, A.; Prasad, J.; Das, S.; Dwivedy, A.K. Essential Oils and Their Application in Food Safety. Front. Sustain. Food Syst. 2021, 5, 133. [CrossRef]

2. Amorati, R.; Foti, M.C.; Valgimigli, L. Antioxidant activity of essential oils. J. Agric. Food Chem. 2013, 61, 10835-10847. [CrossRef] [PubMed]

3. Sharma, S.; Barkauskaite, S.; Jaiswal, A.K.; Jaiswal, S. Essential oils as additives in active food packaging. Food Chem. 2021, 343, 128403. [CrossRef]

4. Do Nascimento, L.D.; de Moraes, A.A.B.; da Costa, K.S.; Galúcio, J.M.P.; Taube, P.S.; Costa, C.M.L.; Cruz, J.N.; de Aguiar Andrade, E.H.; de Faria, L.J.G. Bioactive natural compounds and antioxidant activity of essential oils from spice plants: New findings and potential applications. Biomolecules 2020, 10, 988. [CrossRef]

5. Vianna, T.C.; Marinho, C.O.; Marangoni Júnior, L.; Ibrahim, S.A.; Vieira, R.P. Essential oils as additives in active starch-based food packaging films: A review. Int. J. Biol. Macromol. 2021, 182, 1803-1819. [CrossRef] [PubMed]

6. del Carmen Razola-Díaz, M.; Guerra-Hernández, E.J.; García-Villanova, B.; Verardo, V. Recent developments in extraction and encapsulation techniques of orange essential oil. Food Chem. 2021, 354, 129575. [CrossRef] [PubMed]

7. Brand-Williams, W.; Cuvelier, M.E.; Berset, C. Use of a free redical method to evaluate antioxidant activity. LWT-Food Sci. Technol. 1995, 28, 25-30. [CrossRef]

8. Parejo, I.; Codina, C.; Petrakis, C.; Kefalas, P. Evaluation of scavenging activity assessed by Co(II)/EDTA-induced luminol chemiluminescence and DPPH·(2,2-diphenyl-1-picrylhydrazyl) free radical assay. J. Pharmacol. Toxicol. Methods 2000, 44, 507-512. [CrossRef]

9. Re, R.; Pellegrini, N.; Proteggente, A.; Pannala, A.; Yang, M.; Rice-Evans, C. Antioxidant activity applying an improved ABTS radical cation decolorization assay. Free Radic. Biol. Med. 1999, 26, 1231-1237. [CrossRef]

10. Hayes, A.J.; Markovic, B. Toxicity of Australian essential oil Backhousia citriodora (Lemon myrtle). Part 1. Antimicrobial activity and in vitro cytotoxicity. Food Chem. Toxicol. 2002, 40, 535-543. [CrossRef]

11. Schneider, C.A.; Rasband, W.S.; Eliceiri, K.W. NIH Image to ImageJ: 25 years of image analysis. Nat. Methods 2012, 9, 671-675. [CrossRef] [PubMed]

12. Ben Lajnef, H.; Ferioli, F.; Pasini, F.; Politowicz, J.; Khaldi, A.; D’Antuono, L.F.; Caboni, M.F.; Nasri, N. Chemical composition and antioxidant activity of the volatile fraction extracted from air-dried fruits of Tunisian Eryngium maritimum L. ecotypes. J. Sci. Food Agric. 2018, 98, 635-643. [CrossRef] [PubMed]

13. Ribeiro-Santos, R.; Andrade, M.; Madella, D.; Martinazzo, A.P.; de Aquino Garcia Moura, L.; de Melo, N.R.; Sanches-Silva, A. Revisiting an ancient spice with medicinal purposes: Cinnamon. Trends Food Sci. Technol. 2017, 62, 154-169. [CrossRef]

14. Porres-Martínez, M.; González-Burgos, E.; Carretero, M.E.; Gómez-Serranillos, M.P. Influence of phenological stage on chemical composition and antioxidant activity of Salvia lavandulifolia Vahl. essential oils. Ind. Crops Prod. 2014, 53, 71-77. [CrossRef]

15. Zorga, J.; Kunicka-Styczynska, A.; Gruska, R.; Smigielski, K. Ultrasound-Assisted Hydrodistillation of Essential Oil from Celery Seeds (Apium graveolens L.) and Its Biological and Aroma Profile. Molecules 2020, 25, 5322. [CrossRef] [PubMed]

16. Stan, M.; Soran, M.L.; Varodi, C.; Lung, I.; Copolovici, L.; Măruţoiu, C. Extraction and GC determination of volatile aroma compounds from extracts of three plant species of the Apiaceae family. AIP Conf. Proc. 2013, 1565, 75-78.

17. Singh, B.; Singh, J.P.; Kaur, A.; Yadav, M.P. Insights into the chemical composition and bioactivities of citrus peel essential oils. Food Res. Int. 2021, 143, 110231. [CrossRef] [PubMed]

18. Ferioli, F.; Giambanelli, E.; D’Antuono, L.F. Fennel (Foeniculum vulgare Mill. subsp. piperitum) florets, a traditional culinary spice in Italy: Evaluation of phenolics and volatiles in local populations, and comparison with the composition of other plant parts. J. Sci. Food Agric. 2017, 97, 5369-5380. [CrossRef] [PubMed]

19. Heer, A.; Guleria, S.; Razdan, V.K. Chemical composition, antioxidant and antimicrobial activities and characterization of bioactive compounds from essential oil of Cinnamomum tamala grown in north-western Himalaya. J. Plant Biochem. Biotechnol. 2017, 26, 191-198. [CrossRef]

20. Salleh, W.M.N.H.W.; Ahmad, F.; Yen, K.H. Antioxidant and anticholinesterase activities of essential oils of cinnamomum griffithii and C. macrocarpum. Nat. Prod. Commun. 2015, 10, 1465-1468. [CrossRef]

21. Kumar, B.H.; Shani, B. Haseena Antioxidant potential and antimicrobial activity of Cinnamomum malabathrum (Batka). Orient. J. Chem. 2010, 26, 1449-1453.

22. Sriramavaratharajan, V.; Murugan, R. Chemical profile of leaf essential oil of cinnamomum walaiwarense and comparison of its antioxidant and hypoglycemic activities with the major constituent benzyl benzoate. Nat. Prod. Commun. 2018, 13, 779-782. [CrossRef]

23. Gogoi, R.; Sarma, N.; Loying, R.; Pandey, S.K.; Begum, T.; Lal, M. A Comparative Analysis of Bark and Leaf Essential Oil and their Chemical Composition, Antioxidant, Anti-inflammatory, Antimicrobial Activities and Genotoxicity of North East Indian Cinnamomum zeylanicum Blume. Nat. Prod. J. 2021, 11, 74-84.

24. Abdelwahab, S.I.; Mariod, A.A.; Taha, M.M.E.; Zaman, F.Q.; Abdelmageed, A.H.A.; Khamis, S.; Sivasothy, Y.; Awang, K. Chemical composition and antioxidant properties of the essential oil of Cinnamomum altissimum Kosterm. (Lauraceae). Arab. J. Chem. 2017, 10, 131-135. [CrossRef] 
25. Foudah, A.I.; Shakeel, F.; Alqarni, M.H.; Ross, S.A.; Salkini, M.A.; Alam, P. Simultaneous Estimation of Cinnamaldehyde and Eugenol in Essential Oils and Traditional and Ultrasound-Assisted Extracts of Different Species of Cinnamon Using a Sustainable/Green HPTLC Technique. Molecules 2021, 26, 2054. [CrossRef] [PubMed]

26. Hamada, H.; Al-Waili, N.; Aboulghazi, A.; Abdellaoui, A.; Al-Waili, T.; Lyoussi, B. Chemical composition and antioxidant content of Thymus vulgaris honey and Origanum vulgare essential oil; their effect on carbon tetrachlorideinduced toxicity. Vet. World 2021, 14, 292-301.

27. Sokmen, A.; Abdel-Baki, A.A.S.; Al-Malki, E.S.; Al-Quraishy, S.; Abdel-Haleem, H.M. Constituents of essential oil of Origanum minutiflorum and its in vitro antioxidant, scolicidal and anticancer activities. J. King Saud Univ.-Sci. 2020, 32, $2377-2382$. [CrossRef]

28. Boskovic, M.; Glisic, M.; Djordjevic, J.; Starcevic, M.; Glamoclija, N.; Djordjevic, V.; Baltic, M.Z. Antioxidative Activity of Thyme (Thymus vulgaris) and Oregano (Origanum vulgare) Essential Oils and Their Effect on Oxidative Stability of Minced Pork Packaged Under Vacuum and Modified Atmosphere. J. Food Sci. 2019, 84, 2467-2474. [CrossRef]

29. Sokmen, A.; Gulluce, M.; Akpulat, H.A.; Daferera, D.; Tepe, B.; Polissiou, M.; Sokmen, M.; Sahin, F. The in vitro antimicrobial and antioxidant activities of the essential oils and methanol extracts of endemic Thymus spathulifolius. Food Control 2004, 15, 627-634. [CrossRef]

30. Gedikoğlu, A.; Sökmen, M.; Çivit, A. Evaluation of Thymus vulgaris and Thymbra spicata essential oils and plant extracts for chemical composition, antioxidant, and antimicrobial properties. Food Sci. Nutr. 2019, 7, 1704-1714. [CrossRef] [PubMed]

31. Aazza, S.; Lyoussi, B.; Miguel, M.G. Antioxidant and antiacetylcholinesterase activities of some commercial essential oils and their major compounds. Molecules 2011, 16, 7672-7690. [CrossRef] [PubMed]

32. Bouyahya, A.; Et-Touys, A.; Bakri, Y.; Talbaui, A.; Fellah, H.; Abrini, J.; Dakka, N. Chemical composition of Mentha pulegium and Rosmarinus officinalis essential oils and their antileishmanial, antibacterial and antioxidant activities. Microb. Pathog. 2017, 111, 41-49. [CrossRef] [PubMed]

33. Chraibi, M.; Farah, A.; Elamin, O.; Iraqui, H.; Fikri-Benbrahim, K. Characterization, antioxidant, antimycobacterial, antimicrobial effcts of Moroccan rosemary essential oil, and its synergistic antimicrobial potential with carvacrol. J. Adv. Pharm. Technol. Res. 2020, 11, 25-29.

34. Leporini, M.; Bonesi, M.; Loizzo, M.R.; Passalacqua, N.G.; Tundis, R. The essential oil of salvia rosmarinus spenn. From Italy as a source of health-promoting compounds: Chemical profile and antioxidant and cholinesterase inhibitory activity. Plants 2020, 9, 798. [CrossRef]

35. Lakehal, S.; Chaouia, C.; Benrebiha, F.Z. Antibacterial and Antioxidant Activities of Rosemary (Rosmarinus officinalis L.) Essential Oil Growing in Djelfa (Algeria). In Recent Advances in Environmental Science from the Euro-Mediterranean and Surrounding Regions, Vols I and II; Kallel, A., Ksibi, M., BenDhia, H., Khelifi, N., Eds.; Springer International Publishing AG: Cham, Switzerland, 2018; pp. 1253-1254.

36. Bajalan, I.; Rouzbahani, R.; Pirbalouti, A.G.; Maggi, F. Antioxidant and antibacterial activities of the essential oils obtained from seven Iranian populations of Rosmarinus officinalis. Ind. Crops Prod. 2017, 107, 305-311. [CrossRef]

37. Risaliti, L.; Kehagia, A.; Daoultzi, E.; Lazari, D.; Bergonzi, M.C.; Vergkizi-Nikolakaki, S.; Hadjipavlou-Litina, D.; Bilia, A.R. Liposomes loaded with Salvia triloba and Rosmarinus officinalis essential oils: In Vitro assessment of antioxidant, antiinflammatory and antibacterial activities. J. Drug Deliv. Sci. Technol. 2019, 51, 493-498. [CrossRef]

38. Wu, Z.; Tan, B.; Liu, Y.; Dunn, J.; Martorell Guerola, P.; Tortajada, M.; Cao, Z.; Ji, P. Chemical Composition and Antioxidant Properties of Essential Oils from Peppermint, Native Spearmint and Scotch Spearmint. Molecules 2019, 24, 2825. [CrossRef] [PubMed]

39. Fatemi, F.; Dini, S.; Rezaei, M.B.; Dadkhah, A.; Dabbagh, R.; Naij, S. The effect of $\gamma$-irradiation on the chemical composition and antioxidant activities of peppermint essential oil and extract. J. Essent. Oil Res. 2014, 26, 97-104. [CrossRef]

40. Stanojevic, L.P.; Stanojevic, J.S.; Savic, V.L.; Cvetkovic, D.J.; Kolarevic, A.; Marjanovic-Balaban, Z.; Nikolic, L.B. Peppermint and Basil Essential Oils: Chemical Composition, in vitro Antioxidant Activity and in vivo Estimation of Skin Irritation. J. Essent. Oil-Bearing Plants 2019, 22, 979-993. [CrossRef]

41. El Jery, A.; Hasan, M.; Rashid, M.M.; Al Mesfer, M.K.; Danish, M.; Ben Rebah, F. Phytochemical characterization, and antioxidant and antimicrobial activities of essential oil from leaves of the common sage Salvia officinalis L. from Abha, Saudi Arabia. Asian Biomed. 2020, 14, 261-270. [CrossRef]

42. El Euch, S.K.; Hassine, D.B.; Cazaux, S.; Bouzouita, N.; Bouajila, J. Salvia officinalis essential oil: Chemical analysis and evaluation of anti-enzymatic and antioxidant bioactivities. S. Afr. J. Bot. 2019, 120, 253-260. [CrossRef]

43. Fang, S.P.; Xing, X.; Lai, P.X.; Huang, J.J. Chemical Composition and Antioxidant Activity of the Essential Oil from Salvia kiangsiensis. Chem. Nat. Compd. 2018, 54, 591-592. [CrossRef]

44. Hassanen, N.H.; Eissa, A.M.F.; Hafez, S.A.M.; Mosa, E.A. Antioxidant and antimicrobial activity of celery (Apium graveolens) and coriander (Coriandrum sativum) herb and seed essential oils. Int. J. Curr. Microbiol. Appl. Sci. 2015, 4, $284-296$.

45. Kamal, G.M.; Ashraf, M.Y.; Hussain, A.I.; Shahzadi, A.; Chughtai, M.I. Antioxidant potential of peel essential oils of three Pakistani Citrus species: Citrus reticulata, Citrus sinensis, and Citrus paradisii. Pak. J. Bot. 2013, 45, 1449-1454.

46. Phi, N.T.L.; Van Hung, P.; Chi, P.T.L.; Dung, N.H. Impact of Extraction Methods on Antioxidant and Antimicrobial Activities of Citrus Essential Oils. J. Essent. Oil Bear. Plants 2015, 18, 806-817. [CrossRef] 
47. Chi, P.T.L.; Van Hung, P.; Le Thanh, H.; Phi, N.T.L. Valorization of Citrus Leaves: Chemical Composition, Antioxidant and Antibacterial Activities of Essential Oils. Waste Biomass Valorization 2020, 11, 4849-4857. [CrossRef]

48. Kaanin-Boudraa, G.; Brahmi, F.; Wrona, M.; Nerín, C.; Hadjal, S.; Madani, K.; Boulekbache-Makhlouf, L. Citrus $\times$ paradisi essential oil as a promising agent for margarine storage stability: Composition and antioxidant capacity. J. Food Process. Preserv. 2021, 45, e15374. [CrossRef]

49. Ou, M.C.; Liu, Y.H.; Sun, Y.W.; Chan, C.F. The Composition, Antioxidant and Antibacterial Activities of Cold-Pressed and Distilled Essential Oils of Citrus paradisi and Citrus grandis (L.) Osbeck. Evid.-Based Complement. Altern. Med. 2015, $2015,804091$. [CrossRef]

50. Ben Miri, Y.; Arino, A.; Djenane, D. Study of Antifungal, Anti-aflatoxigenic, Antioxidant Activity and Phytotoxicity of Algerian Citrus limon var. Eureka and Citrus sinensis var. Valencia Essential oils. J. Essent. Oil-Bearing Plants 2018, 21, 345-361. [CrossRef]

51. Guo, J.J.; Gao, Z.P.; Xia, J.L.; Ritenour, M.A.; Li, G.Y.; Shan, Y. Comparative analysis of chemical composition, antimicrobial and antioxidant activity of citrus essential oils from the main cultivated varieties in China. Lwt 2018, 97, 825-839. [CrossRef]

52. Fancello, F.; Petretto, G.L.; Zara, S.; Sanna, M.L.; Addis, R.; Maldini, M.; Foddai, M.; Rourke, J.P.; Chessa, M.; Pintore, G. Chemical characterization, antioxidant capacity and antimicrobial activity against food related microorganisms of Citrus limon var. pompia leaf essential oil. LWT-Food Sci. Technol. 2016, 69, 579-585. [CrossRef]

53. Kalleli, F.; Bettaieb Rebey, I.; Wannes, W.A.; Boughalleb, F.; Hammami, M.; Saidani Tounsi, M.; M’hamdi, M. Chemical composition and antioxidant potential of essential oil and methanol extract from Tunisian and French fennel (Foeniculum vulgare Mill.) seeds. J. Food Biochem. 2019, 43, e12935. [CrossRef]

54. Ahmed, A.F.; Shi, M.; Liu, C.; Kang, W. Comparative analysis of antioxidant activities of essential oils and extracts of fennel (Foeniculum vulgare Mill.)seeds from Egypt and China. Food Sci. Hum. Wellness 2019, 8, 67-72. [CrossRef]

55. Semeniuc, C.A.; Pop, C.R.; Rotar, A.M. Antibacterial activity and interactions of plant essential oil combinations against Grampositive and Gram-negative bacteria. J. Food Drug Anal. 2017, 25, 403-408. [CrossRef] [PubMed]

56. Mith, H.; Duré, R.; Delcenserie, V.; Zhiri, A.; Daube, G.; Clinquart, A. Antimicrobial activities of commercial essential oils and their components against food-borne pathogens and food spoilage bacteria. Food Sci. Nutr. 2014, 2, 403-416. [CrossRef] [PubMed]

57. Gucwa, K.; Milewski, S.; Dymerski, T.; Szweda, P. Investigation of the antifungal activity and mode of action of thymus vulgaris, citrus limonum, pelargonium graveolens, cinnamomum cassia, ocimum basilicum, and eugenia caryophyllus essential oils. Molecules 2018, 23, 1116. [CrossRef] [PubMed]

58. Firmino, D.F.; Cavalcante, T.T.A.; Gomes, G.A.; Firmino, N.C.S.; Rosa, L.D.; De Carvalho, M.G.; Catunda, F.E.A. Antibacterial and Antibiofilm Activities of Cinnamomum Sp. Essential Oil and Cinnamaldehyde: Antimicrobial Activities. Sci. World J. 2018, 2018, 7405736. [CrossRef] [PubMed]

59. Vasconcelos, N.G.; Croda, J.; Simionatto, S. Antibacterial mechanisms of cinnamon and its constituents: A review. Microb. Pathog. 2018, 120, 198-203. [CrossRef] [PubMed]

60. Ye, H.; Shen, S.; Xu, J.; Lin, S.; Yuan, Y.; Jones, G.S. Synergistic interactions of cinnamaldehyde in combination with carvacrol against food-borne bacteria. Food Control 2013, 34, 619-623. [CrossRef]

61. Friedman, M. Chemistry, Antimicrobial Mechanisms, and Antibiotic Activities of Cinnamaldehyde against Pathogenic Bacteria in Animal Feeds and Human Foods. J. Agric. Food Chem. 2017, 65, 10406-10423. [CrossRef]

62. Utchariyakiat, I.; Surassmo, S.; Jaturanpinyo, M.; Khuntayaporn, P.; Chomnawang, M.T. Efficacy of cinnamon bark oil and cinnamaldehyde on anti-multidrug resistant Pseudomonas aeruginosa and the synergistic effects in combination with other antimicrobial agents. BMC Complement. Altern. Med. 2016, 16, 158. [CrossRef] [PubMed]

63. Nazzaro, F.; Fratianni, F.; De Martino, L.; Coppola, R.; De Feo, V. Effect of essential oils on pathogenic bacteria. Pharmaceuticals 2013, 6, 1451-1474. [CrossRef] [PubMed]

64. McCabe-Sellers, B.J.; Beattie, S.E. Food safety: Emerging trends in foodborne illness surveillance and prevention. J. Am. Diet. Assoc. 2004, 104, 1708-1717. [CrossRef]

65. Sergelidis, D.; Angelidis, A.S. Methicillin-resistant Staphylococcus aureus: A controversial food-borne pathogen. Lett. Appl. Microbiol. 2017, 64, 409-418. [CrossRef] [PubMed]

66. Silva, N.; Alves, S.; Gonçalves, A.; Amaral, J.S.; Poeta, P. Antimicrobial activity of essential oils from mediterranean aromatic plants against several foodborne and spoilage bacteria. Food Sci. Technol. Int. 2013, 19, 503-510. [CrossRef] [PubMed]

67. Bozin, B.; Mimica-Dukic, N.; Simin, N.; Anackov, G. Characterization of the volatile composition of essential oils of some lamiaceae spices and the antimicrobial and antioxidant activities of the entire oils. J. Agric. Food Chem. 2006, 54, 1822-1828. [CrossRef]

68. Niu, C.; Wang, C.; Yang, Y.; Chen, R.; Zhang, J.; Chen, H.; Zhuge, Y.; Li, J.; Cheng, J.; Xu, K.; et al. Carvacrol Induces Candida albicans Apoptosis Associated With $\mathrm{Ca}^{2+} /$ Calcineurin Pathway. Front. Cell. Infect. Microbiol. 2020, 10, 1-12. [CrossRef] [PubMed]

69. Ben Arfa, A.; Combes, S.; Preziosi-Belloy, L.; Gontard, N.; Chalier, P. Antimicrobial activity of carvacrol related to its chemical structure. Lett. Appl. Microbiol. 2006, 43, 149-154. [CrossRef] [PubMed]

70. Lambert, R.J.W.; Skandamis, P.N.; Coote, P.J.; Nychas, G.J.E. A study of the minimum inhibitory concentration and mode of action of oregano essential oil, thymol and carvacrol. J. Appl. Microbiol. 2001, 91, 453-462. [CrossRef] [PubMed]

71. Bagamboula, C.F.; Uyttendaele, M.; Debevere, J. Inhibitory effect of thyme and basil essential oils, carvacrol, thymol, estragol, linalool and p-cymene towards Shigella sonnei and S. flexneri. Food Microbiol. 2004, 21, 33-42. [CrossRef] 
72. Cosentino, S.; Tuberoso, C.I.G.; Pisano, B.; Satta, M.; Mascia, V.; Arzedi, E.; Palmas, F. In-Vitro antimicrobial activity and chemical composition of Sardinian Thymus essential oils. Lett. Appl. Microbiol. 1999, 29, 130-135. [CrossRef]

73. Cristani, M.; D'Arrigo, M.; Mandalari, G.; Castelli, F.; Sarpietro, M.G.; Micieli, D.; Venuti, V.; Bisignano, G.; Saija, A.; Trombetta, D. Interaction of four monoterpenes contained in essential oils with model membranes: Implications for their antibacterial activity. $J$. Agric. Food Chem. 2007, 55, 6300-6308. [CrossRef] [PubMed]

74. Ebani, V.V.; Nardoni, S.; Bertelloni, F.; Tosi, G.; Massi, P.; Pistelli, L.; Mancianti, F. In Vitro antimicrobial activity of essential oils against salmonella enterica serotypes enteritidis and typhimurium strains isolated from poultry. Molecules 2019, 24, 900. [CrossRef]

75. Prabuseenivasan, S.; Jayakumar, M.; Ignacimuthu, S. In Vitro antibacterial activity of some plant essential oils. BMC Complement. Altern. Med. 2006, 6, 39. [CrossRef] [PubMed]

76. Brnawi, W.I.; Hettiarachchy, N.S.; Horax, R.; Kumar-Phillips, G.; Ricke, S. Antimicrobial activity of leaf and bark cinnamon essential oils against Listeria monocytogenes and Salmonella typhimurium in broth system and on celery. J. Food Process. Preserv. 2019, 43, e13888. [CrossRef]

77. Blaszyk, M.; Holley, R.A. Interaction of monolaurin, eugenol and sodium citrate on growth of common meat spoilage and pathogenic organisms. Int. J. Food Microbiol. 1998, 39, 175-183. [CrossRef]

78. Zhang, L.L.; Zhang, L.F.; Xu, J.G.; Hu, Q.P. Comparison study on antioxidant, DNA damage protective and antibacterial activities of eugenol and isoeugenol against several foodborne pathogens. Food Nutr. Res. 2017, 61, 1353356. [CrossRef]

79. Dorman, H.J.D.; Deans, S.G. Antimicrobial agents from plants: Antibacterial activity of plant volatile oils. J. Appl. Microbiol. 2000, 88, 308-316. [CrossRef] [PubMed]

80. Hou, H.; Zhang, X.; Zhao, T.; Zhou, L. Effects of Origanum vulgare essential oil and its two main components, carvacrol and thymol, on the plant pathogen Botrytis cinerea. PeerJ 2020, 8, e9626. [CrossRef] [PubMed]

81. El-Mogy, M.M.; Alsanius, B.W. Cassia oil for controlling plant and human pathogens on fresh strawberries. Food Control 2012, 28, 157-162. [CrossRef]

82. Soylu, E.M.; Kurt, Ş.; Soylu, S. In Vitro and in vivo antifungal activities of the essential oils of various plants against tomato grey mould disease agent Botrytis cinerea. Int. J. Food Microbiol. 2010, 143, 183-189. [CrossRef]

83. De Clerck, C.; Dal Maso, S.; Parisi, O.; Dresen, F.; Zhiri, A.; Haissam Jijakli, M. Screening of Antifungal and Antibacterial Activity of 90 Commercial Essential Oils against 10 Pathogens of Agronomical Importance. Foods 2020, 9, 1418. [CrossRef] [PubMed]

84. Palfi, M.; Konjevoda, P.; Vrandečić, K.; Ćosić, J. Antifungal activity of essential oils on mycelial growth of Fusarium oxysporum and Bortytis cinerea. Emir. J. Food Agric. 2019, 31, 544-554. [CrossRef]

85. Grul'ová, D.; Caputo, L.; Elshafie, H.S.; Baranová, B.; de Martino, L.; Sedlák, V.; Gogal'ová, Z.; Poráčová, J.; Camele, I.; de Feo, V. Thymol Chemotype Origanum vulgare L. Essential oil as a potential selective bio-based herbicide on monocot plant species. Molecules 2020, 25, 595. [CrossRef] [PubMed]

86. Williamson, B.; Tudzynski, B.; Tudzynski, P.; Van Kan, J.A.L. Botrytis cinerea: The cause of grey mould disease. Mol. Plant Pathol. 2007, 8, 561-580. [CrossRef] [PubMed]

87. Ahmadu, T.; Ahmad, K.; Ismail, S.I.; Rashed, O.; Asib, N.; Omar, D. Antifungal efficacy of moringa oleifera leaf and seed extracts against botrytis cinerea causing gray mold disease of tomato (Solanum lycopersicum L.). Braz. J. Biol. 2021, 81, 1007-1022. [CrossRef] [PubMed]

88. Leroux, P. Chemical Control of Botrytis and its Resistance to Chemical Fungicides. In Botrytis: Biology, Pathology and Control; Elad, Y., Williamson, B., Tudzynski, P., Delen, N., Eds.; Springer: Dordrecht, The Netherlands, 2007; pp. $195-222$.

89. Leroux, P.; Fritz, R.; Debieu, D.; Albertini, C.; Lanen, C.; Bach, J.; Gredt, M.; Chapeland, F. Mechanisms of resistance to fungicides in field strains of B. cinerea. Pest Manag. Sci. 2002, 58, 876-888. [CrossRef]

90. Leroux, P.; Gredt, M.; Leroch, M.; Walker, A.S. Exploring mechanisms of resistance to respiratory inhibitors in field strains of botrytis cinerea, the causal agent of gray mold. Appl. Environ. Microbiol. 2010, 76, 6615-6630. [CrossRef]

91. Bardas, G.A.; Veloukas, T.; Koutita, O.; Karaoglanidis, G.S. Multiple resistance of Botrytis cinerea from kiwifruit to SDHIs, QoIs and fungicides of other chemical groups. Pest Manag. Sci. 2010, 66, 967-973. [CrossRef]

92. Raveau, R.; Fontaine, J.; Lounès-Hadj Sahraoui, A. Essential oils as potential alternative biocontrol products against plant pathogens and weeds: A review. Foods 2020, 9, 365. [CrossRef] [PubMed]

93. Mani-López, E.; Cortés-Zavaleta, O.; López-Malo, A. A review of the methods used to determine the target site or the mechanism of action of essential oils and their components against fungi. SN Appl. Sci. 2021, 3, 44. [CrossRef]

94. Akthar, M.S.; Degaga, B.; Azam, T. Antimicrobial activity of essential oils extracted from medicinal plants against the pathogenic microorganisms: A review. Issues Biol. Sci. Pharm. Res. 2014, 2, 001-007.

95. Valdivieso-Ugarte, M.; Gomez-Llorente, C.; Plaza-Díaz, J.; Gil, Á. Antimicrobial, antioxidant, and immunomodulatory properties of essential oils: A systematic review. Nutrients 2019, 11, 2786. [CrossRef] [PubMed]

96. Wink, M. Modes of Action of Herbal Medicines and Plant Secondary Metabolites. Medicines 2015, 2, 251-286. [CrossRef] [PubMed] 